\title{
Capturing Complex Behavior in Josephson Traveling-Wave Parametric Amplifiers
}

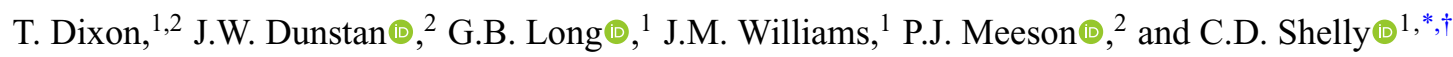 \\ ${ }^{1}$ National Physical Laboratory, Hampton Road, Teddington TW11 0LW, United Kingdom \\ ${ }^{2}$ Royal Holloway University of London, Egham, Surrey TW20 OEX, United Kingdom
}

(Received 6 December 2019; revised 16 July 2020; accepted 21 August 2020; published 22 September 2020)

\begin{abstract}
We present an analysis of wave mixing in the recently developed Josephson traveling-wave parametric amplifier (JTWPA). Circuit simulations performed using WRSPICE show the full behavior of the JTWPA, allowing propagation of all tones. The coupled mode equations (CMEs) containing only pump, signal, and idler propagation are shown to be insufficient to completely capture complex mixing behavior in the JTWPA. Extension of the CMEs through additional state vectors in the analytic solutions allows closer agreement with WRSPICE. We consider an ordered framework for the systematic inclusion of extended eigenmodes and make a comparison with WRSPICE at each step. The agreement between the two methods validates both approaches and provides insight into the operation of the JTWPA.
\end{abstract}

DOI: 10.1103/PhysRevApplied.14.034058

\section{INTRODUCTION}

Josephson-junction (JJ)-based parametric amplifiers (JPAs) [1-3] have been used in recent years to provide quantum-limited noise performance for quantum optics experiments [4], single-microwave-photon detection [5], high-fidelity qubit readout for quantum information technologies [6,7], and producing squeezed states [8]. These microwave small-signal amplifiers have been shown to exhibit large gain (greater than $20 \mathrm{~dB}$ ) $[9,10]$, and approach the quantum noise limit [11]. Typically, these amplifiers have utilized high- $Q$ superconducting resonators, which have a limited bandwidth and dynamic range. Removing the resonant architecture and allowing nonlinear interactions along a transmission line can increase both the dynamic range and the bandwidth [12]. More recently, the Josephson traveling-wave parametric amplifier (JTWPA), based on JJs embedded in a microwave transmission line, has been shown to provide large gain without the bandwidth limitation of JPAs [13-15].

Implementing unbiased JJs along the transmission line leads to centrosymmetry of the system and four-wave mixing $(4 \mathrm{WM})$, whereby the signal and idler frequencies are

\footnotetext{
*cshelly@oxfordquantumcircuits.com

†Present address: Oxford Quantum Circuits, Thames Valley Science Park, Shinfield, Reading, RG2 9LH, UK.

Published by the American Physical Society under the terms of the Creative Commons Attribution 4.0 International license. Further distribution of this work must maintain attribution to the author(s) and the published article's title, journal citation, and DOI.
}

close to the frequency of the pump, i.e., $f_{s}+f_{i}=2 f_{p}$. In this paper, we focus primarily on the three-wave mixing (3WM) scheme, $f_{s}+f_{i}=f_{p}$, which shifts the pump frequency away from that of the signal and idler, allowing the pump to be filtered more easily from the signal. The 3WM regime also takes advantage of the inherently stronger interactions than in the $4 \mathrm{WM}$ regime. In this regime, the phase-modulation effect and the signal gain are controlled independently, the process of which was described in detail by Zorin [16].

To access the $3 \mathrm{WM}$ regime, rf superconducting quantum interference devices ( $r f$ SQUIDs) are embedded in the transmission line. The current-phase relation of a $\mathrm{rf}$ SQUID is given by [16]

$$
I(\varphi)=I_{c}\left[\frac{\varphi}{\beta_{L}}+\sin \left(\varphi_{\mathrm{dc}}+\varphi\right)-\sin \left(\varphi_{\mathrm{dc}}\right)\right] .
$$

By expansion of the $\varphi$ term, we can describe the current $I(\varphi)$ by the formula

$$
I(\varphi)=I_{c}\left[\frac{\varphi}{\beta_{L}}-\sin \left(\varphi_{\mathrm{dc}}\right)\left(\frac{\varphi^{2}}{2}\right)+\cos \left(\varphi_{\mathrm{dc}}\right)\left(\varphi-\frac{\varphi^{3}}{6}\right)\right] .
$$

For $\varphi_{\mathrm{dc}}=0, n \pi$, Eq. (2) is centrosymmetric $[I(-\varphi)=$ $-I(\varphi)]$ and is in the purely $4 \mathrm{WM}$ regime. If the dc bias $\varphi_{\mathrm{dc}}$ is varied away from $0, n \pi$, Eq. (2) becomes noncentrosymmetric $[I(-\varphi) \neq-I(\varphi)]$. When $\varphi_{\mathrm{dc}}=(n+1 / 2) \pi$, the cubic term vanishes, resulting in a predominantly $3 \mathrm{WM}$ regime. The circuit as proposed in Ref. [16] is shown in Fig. 1, and the variation of $\varphi_{\mathrm{dc}}$ can be achieved either by 


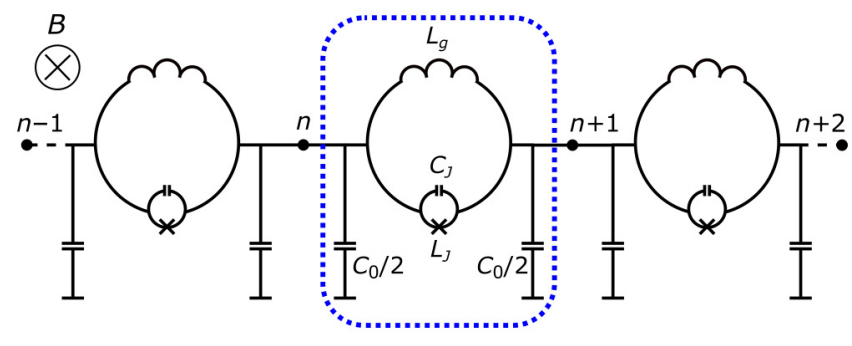

FIG. 1. Circuit schematic showing three cells of an $N$-cell array of rf SQUIDs implemented in a microwave transmissionline design as proposed by Zorin [16]. The blue dotted line highlights one of the repeating cell elements. An externally applied magnetic field is used to shift the operation of the JTWPA to the $3 \mathrm{WM}$ regime (in the WRSPICE simulations presented here, flux coupling to the rf SQUID is used to bias the JTWPA). Each cell consists of a rf SQUID with geometric inductance $L_{g}$, and a Josephson junction with Josephson inductance $L_{J}$ and junction capacitance $C_{J}$. Each cell has a capacitance to ground $C_{0}$. The parameters of the circuit presented in this paper are $I_{c}=5 \mu \mathrm{A}$, $C_{J}=60 \mathrm{fF}, C_{0}=100 \mathrm{fF}$, and $L_{g}=57 \mathrm{pH}$.

applying an external magnetic field to the SQUIDs or by applying a dc current along the transmission line.

\section{MODELING THE JTWPA}

\section{A. WRSPICE simulations}

In order to capture the full behavior of the JTWPA, we use WRSPICE to simulate the circuit design shown in Fig. 1. WRSPICE is a SPICE-like circuit simulator that includes a Josephson-junction model [17]. Conventional analytical models describing three-wave mixers consider only three mixing tones, the pump $f_{p}$, signal $f_{s}$, and $\operatorname{idler} f_{i}$ [18]. Using WRSPICE, we observe that other mixing tones, especially harmonics of the pump, are generated in the JTWPA. In this paper, we show that generation of other mixing tones leads to a strong reduction in the signal gain that can be achieved. In WRSPICE, we implement a 500-cell version of the circuit shown in Fig. 1. The rf SQUIDs are fluxbiased such that we operate in the $3 \mathrm{WM}$ regime. A strong pump current $\left[I_{p}^{\mathrm{rms}}(0) \approx 1.97 \mu \mathrm{A}, P_{p}(0) \approx-70 \mathrm{dBm}\right.$ for $\left.Z_{0}=23.87 \Omega\right]$ at $f_{p}=12 \mathrm{GHz}$ and a weak signal current $\left[I_{s}^{\mathrm{rms}}(0) \approx 0.10 \mu \mathrm{A}, P_{s}(0) \approx-96 \mathrm{dBm}\right]$ at $f_{s}=7.2 \mathrm{GHz}$ are input into the JTWPA at node 0 . The values replicate those used by Zorin [16] as example parameters in the analytical model. By performing a FFT of the current entering each node $n$, we observe the behavior of all tones propagating along the amplifier. We observe wavemixing processes, including generation of an idler tone $\left(f_{i}=4.8 \mathrm{GHz}\right)$ at the difference between the pump and signal tones. This wave mixing derives solely from the nonlinear current-phase relation of the Josephson junction, $I=I_{c} \sin (\varphi)$, and demonstrates the ability of WRSPICE to model the nonlinear behavior of the system. Figure 2 shows a color map of the current at each node of the

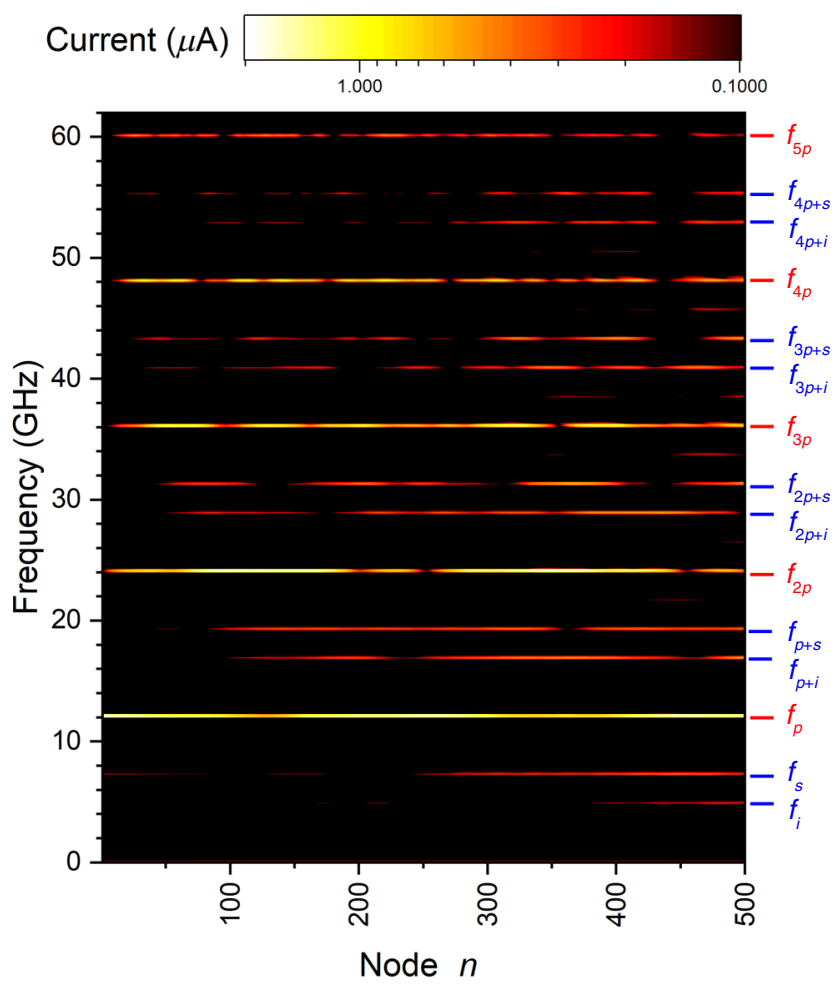

FIG. 2. Color map showing the current at each node of the JTWPA circuit, as simulated in WRSPICE. The color map shows the expected pump, signal, and idler tones as well as the generation of additional tones. The harmonics of the pump (second-, third-, fourth-, and fifth-harmonic generation are shown) are clearly observed. Sum-frequency generation (pump + idler, pump + signal, etc.) is also observed. Pump-harmonic terms are labeled in red, and pump-mediated sum-frequency generation in blue.

JTWPA as simulated by WRSPICE. Note that as well as the signal, pump, and idler, we observe significant generation of pump harmonics $f_{2 p}, f_{3 p}, f_{4 p}$, and $f_{5 p}$. In addition, we observe sum-frequency generation associated with the pump and the pump harmonics.

The signal tone is amplified along the JTWPA from an input amplitude of $I_{s}^{\mathrm{rms}}(0) \approx 0.10 \mu \mathrm{A}$ to $I_{s}^{\mathrm{rms}}(399) \approx$ $0.19 \mu \mathrm{A}$, representing a signal gain of $5.6 \mathrm{~dB}$. This gain is significantly less than that predicted in Ref. [16] for the same pump and signal input amplitudes and JTWPA length. We show here that the generation of the additional terms seen in the WRSPICE simulations accounts for most of the reduction in amplifier gain observed in WRSPICE compared with the gain expected from the analytical theory described in Ref. [16]. It is therefore clear that, for the given circuit parameters, additional tones must be taken into account in the analytical theory.

\section{B. Extension of the coupled mode equations}

To allow the analytical theory to capture more of the behavior demonstrated by the JTWPA simulations, we 
TABLE I. Tones included in each CME set. CME- $K$ contains all pump-mediated mixing tones up to and including the $K$ th harmonic of the pump.

\begin{tabular}{lcccc}
\hline \hline CME-1 & CME-2 & CME-3 & CME-4 & CME-5 \\
\hline$f_{i}$ & $f_{i}$ & $f_{i}$ & $f_{i}$ & $f_{i}$ \\
$f_{s}$ & $f_{s}$ & $f_{s}$ & $f_{s}$ & $f_{s}$ \\
$f_{p}$ & $f_{p}$ & $f_{p}$ & $f_{p}$ & $f_{p}$ \\
& $f_{p+i}$ & $f_{p+i}$ & $f_{p+i}$ & $f_{p+i}$ \\
& $f_{p+s}$ & $f_{p+s}$ & $f_{p+s}$ & $f_{p+s}$ \\
& $f_{2 p}$ & $f_{2 p}$ & $f_{2 p}$ & $f_{2 p}$ \\
& & $f_{2 p+i}$ & $f_{2 p+i}$ & $f_{2 p+i}$ \\
& $f_{2 p+s}$ & $f_{2 p+s}$ & $f_{2 p+s}$ \\
& $f_{3 p}$ & $f_{3 p}$ & $f_{3 p}$ \\
& & $f_{3 p+i}$ & $f_{3 p+i}$ \\
& & $f_{3 p+s}$ & $f_{3 p+s}$ \\
& & $f_{4 p}$ & $f_{4 p}$ \\
& & & $f_{4 p+i}$ \\
& & & $f_{5 p}$ \\
\hline \hline
\end{tabular}

extend the coupled mode equations (CMEs) to include additional tones. The theory extension method is similar to that considered by Chaudhuri et al. for the 4WM case [19]. In Table I, the conventional theory as presented in Ref. [16] is denoted as "CME-1" and includes the pump, signal, and idler tones. Each further CME extension (CME- $K$ ) contains all pump-mediated mixing tones up to and including the $K$ th harmonic of the pump. Here, we extend the theory up to CME-5. The constituent tones of each CME set are shown in Table I.

The inclusion of tones in the extended CMEs is described in detail below for the case of CME-2 (inclusion of the second harmonic of the pump, $f_{2 p}$, and of the pump-mediated sum-frequency generation, $f_{p+i}$ and $f_{p+s}$ ). We introduce additional propagators $\partial A_{p+i} / \partial x, \partial A_{p+s} / \partial x$, and $\partial A_{2 p} / \partial x$ in the allowed space of states $\Phi$, where

$$
\Phi=\frac{1}{2} \sum_{\substack{j=i, s, p, p+i, p+s, 2 p}} A_{j}(x) e^{i\left(k_{j} x-\omega_{j} t\right)}+\text { c.c. }
$$

and where $A_{j}(x)$ is the amplitude at dimensionless coordinate $x$ along the JTWPA of the $j$ th tone in the space of states. Each additional tone is treated as a generated tone in the same way as for the idler, that is, $A_{p+i}(0)=A_{p+s}(0)=$ $A_{2 p}(0)=A_{i}(0)=0$.

For the purely $3 \mathrm{WM}$ regime, the wave equation describing a rf-SQUID-embedded transmission line is given by $[15,16]$

$$
\frac{\partial^{2} \Phi}{\partial x^{2}}-\omega_{0}^{-2} \frac{\partial^{2} \Phi}{\partial t^{2}}+\omega_{J}^{-2} \frac{\partial^{4} \Phi}{\partial x^{2} \partial t^{2}}+\beta \frac{\partial}{\partial x}\left[\left(\frac{\partial \Phi}{\partial x}\right)^{2}\right]=0,
$$

where

$$
\omega_{0}=\frac{1}{\sqrt{L_{g} C_{0}}} \quad \text { and } \quad \omega_{J}=\frac{1}{\sqrt{L_{g} C_{J}}}
$$

and

$$
\beta=\beta_{L} \frac{1}{2} \sin \left(\varphi_{\mathrm{dc}}\right) \quad \text { and } \quad \beta_{L}=\frac{2 \pi L_{g} I_{c}}{\Phi_{0}},
$$

where $\omega_{J}$ is the plasma frequency, $L_{g}$ is the geometric inductance of the SQUID loop, $C_{0}$ is the capacitance to ground of the line, $C_{J}$ is the junction capacitance, $I_{c}$ is the junction critical current, and $\Phi_{0}$ is the magnetic flux quantum. By assuming that the nonlinear component of Eq. (4) acts as a perturbation to the superlinear equation

$$
\frac{\partial^{2} \Phi}{\partial x^{2}}-\omega_{0}^{-2} \frac{\partial^{2} \Phi}{\partial t^{2}}+\omega_{J}^{-2} \frac{\partial^{4} \Phi}{\partial x^{2} \partial t^{2}}=0,
$$

we take the resulting superlinear dispersion solution,

$$
k(\omega)=\frac{\omega}{\omega_{0}\left(\sqrt{1-\omega^{2} / \omega_{J}^{2}}\right)},
$$

and the space of allowed states in Eq. (3) as a trial solution to generate the coupled mode equations CME-2.

For frequencies much lower than the junction plasma frequency, $\omega^{2} / \omega_{J}^{2} \approx 0$, and therefore Eq. (6) can be simplified to $k(\omega) \approx \omega / \omega_{0}$. We now construct a simple set of CMEs including the tones $f_{p+i}, f_{p+s}$, and $f_{2 p}$, to find

$$
\begin{aligned}
\frac{d A_{i}}{d x}= & \frac{\beta}{2}\left(k_{p} k_{s} A_{p} A_{s}^{*} e^{i\left(k_{p}-k_{s}\right) x}+k_{p} k_{p+i} A_{p+i} A_{p}^{*} e^{i\left(k_{p+i}-k_{p}\right) x}+k_{2 p} k_{p+s} A_{2 p} A_{p+s}^{*} e^{i\left(k_{2 p}-k_{p+s}\right) x}\right) e^{-i k_{i} x} \\
\frac{d A_{s}}{d x}= & \frac{\beta}{2}\left(k_{p} k_{i} A_{p} A_{i}^{*} e^{i\left(k_{p}-k_{i}\right) x}+k_{p} k_{p+s} A_{p+s} A_{p}^{*} e^{i\left(k_{p+s}-k_{p}\right) x}+k_{2 p} k_{p+i} A_{2 p} A_{p+i}^{*} e^{i\left(k_{2 p}-k_{p+i}\right) x}\right) e^{-i k_{s} x} \\
\frac{d A_{p}}{d x}= & \frac{\beta}{2}\left(-k_{s} k_{i} A_{i} A_{s} e^{i\left(k_{s}+k_{i}\right) x}+k_{p+s} k_{S} A_{p+s} A_{s}^{*} e^{i\left(k_{p+s}-k_{s}\right) x}+k_{p+i} k_{i} A_{p+i} A_{i}^{*} e^{i\left(k_{p+i}-k_{i}\right) x}\right. \\
& \left.+k_{2 p} k_{p} A_{2 p} A_{p}^{*} e^{i\left(k_{2 p}-k_{p}\right) x}\right) e^{-i k_{p} x}
\end{aligned}
$$




$$
\begin{aligned}
\frac{d A_{p+i}}{d x} & =\frac{\beta}{2}\left(-k_{p} k_{i} A_{p} A_{i} e^{i\left(k_{p}+k_{i}\right) x}+k_{2 p} k_{s} A_{2 p} A_{s}^{*} e^{i\left(k_{2 p}-k_{s}\right) x}\right) e^{-i k_{p+i} x}, \\
\frac{d A_{p+s}}{d x} & =\frac{\beta}{2}\left(-k_{p} k_{s} A_{p} A_{s} e^{i\left(k_{p}+k_{s}\right) x}+k_{2 p} k_{i} A_{2 p} A_{i}^{*} e^{i\left(k_{2 p}-k_{i}\right) x}\right) e^{-i k_{p+s} x}, \\
\frac{d A_{2 p}}{d x} & =\frac{\beta}{2}\left(-\frac{k_{p}^{2} A_{p}^{2}}{2} e^{i\left(k_{p}+k_{p}\right) x}-k_{p+i} k_{s} A_{p+i} A_{s} e^{i\left(k_{p+i}+k_{s}\right) x}-k_{p+s} k_{i} A_{p+s} A_{i} e^{i\left(k_{p}+s+k_{i}\right) x}\right) e^{-i k_{2 p} x} .
\end{aligned}
$$

If we neglect all terms proportional to $A_{p+i}, A_{p+s}$, and $A_{2 p}$, as well as their derivatives, we recover the conventional CMEs used to describe three-wave parametric amplification,

$$
\begin{gathered}
\frac{d A_{i}}{d x}=\frac{\beta}{2}\left(k_{p} k_{s} A_{p} A_{s}^{*} e^{i\left(k_{p}-k_{s}\right) x}\right) e^{-i k_{i} x} \\
\frac{d A_{s}}{d x}=\frac{\beta}{2}\left(k_{p} k_{i} A_{p} A_{i}^{*} e^{i\left(k_{p}-k_{i}\right) x}\right) e^{-i k_{s} x} \\
\frac{d A_{p}}{d x}=-\frac{\beta}{2}\left(k_{s} k_{i} A_{S} A_{i} e^{i\left(k_{s}+k_{i}\right) x}\right) e^{-i k_{p} x} .
\end{gathered}
$$

Similar sets of extended equations are constructed for CME-3, CME-4, and CME-5 (see Appendices E-G for a full list of the equations). Each set of equations, CME1 [Eqs. (13)-(15)], CME-2 [Eqs. (7)-(12)], CME-3 [Eqs. (E1)-(E9)], CME-4 [Eqs. (F1)-(F12)], and CME-5 [Eqs. (G1)-(G15)], is solved numerically using the ode 45 function in MATLAB. The CMEs are solved using the definition for the phase shift per cell of a lumped-element line,

$$
k=\frac{1}{a} \arccos \left(1-\frac{2 \omega^{2}}{\omega_{c}^{2}\left(1-\omega^{2} / \omega_{J}^{2}\right)}\right),
$$

which is discussed in detail in Appendix C. Figure 11, in Appendix $\mathrm{C}$, compares the agreement between the WRSPICE output and the CME-5 solutions when the phase shift per cell described by Eq. (16) above is used, and when the phase shift per cell described by an approximate form that is equivalent to Eq. (6) is used. Using the exact form [Eq. (16)] provides improved agreement over the full length of the amplifier.

\section{Comparison of WRSPICE simulations and coupled-mode-equation solutions}

In order to compare the WRSPICE simulations with the solutions to the coupled mode equations, it is necessary to relate the current $I^{\mathrm{rms}}(n)$ used in WRSPICE to the amplitude
$A(x)$ used in the CMEs with the following relation:

$$
I^{\mathrm{rms}}(n)=\frac{|A(x)|}{\sqrt{2}} \frac{\omega}{\omega_{0}} \frac{I_{c}}{\beta_{L}},
$$

where $I^{\mathrm{rms}}(n)$ is the current at node $n$. To compare the WRSPICE simulation results with the solutions to the CMEs, we focus first on the interaction between the pump, $f_{p}$, and the second harmonic of the pump, $f_{2 p}$. From the WRSPICE output shown in Fig. 2, it is clear that the $f_{2 p}$ tone is of large amplitude, and thus generation of the second harmonic of the pump is a dominant mixing mechanism not accounted for in the CME-1 theory. Figure 3 shows the solution to CME-5 for the $f_{p}$ and $f_{2 p}$ tones compared with the WRSPICE output. The amplitude of both tones is well described by the CME-5 solutions up to node 250 , beyond which the extent of the agreement reduces.

There are a number of assumptions made in the original CME-1 theory (and carried through into our CME extensions), which are now considered to ensure that we are performing the WRSPICE simulations in a regime in which these assumptions are broadly satisfied. The phase of the junction is set by a dc bias to $\varphi_{\mathrm{dc}}=\pi / 2$ in order to operate in a purely $3 \mathrm{WM}$ regime. The ac phase $\varphi_{\mathrm{ac}}$ is assumed to be small with respect to $\varphi_{\mathrm{dc}}$. Figure 4 shows that for

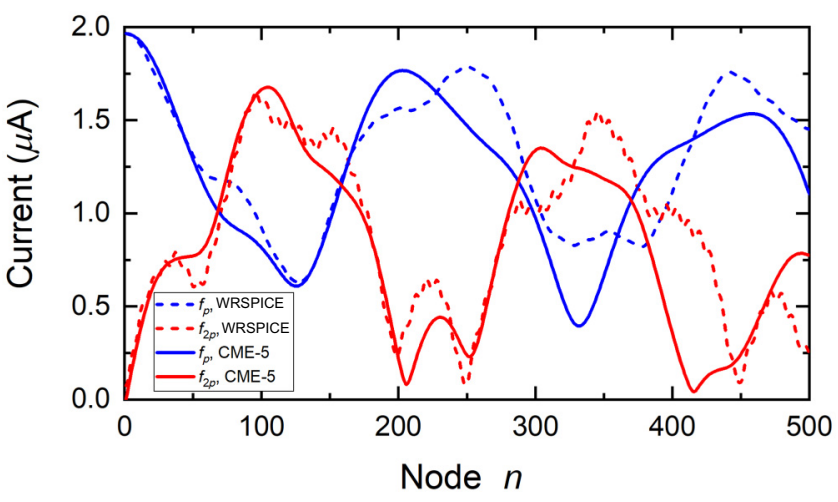

FIG. 3. Comparison between the extended CME-5 and WRSPICE simulations of the pump $\left(f_{p}\right)$ and second harmonic of the pump $\left(f_{2 p}\right) . I_{p}^{\mathrm{rms}}(0) \approx 1.97 \mu \mathrm{A}$. The amplitudes of both tones measured at each node as simulated in WRSPICE are well described using CME-5 up to node 250. 


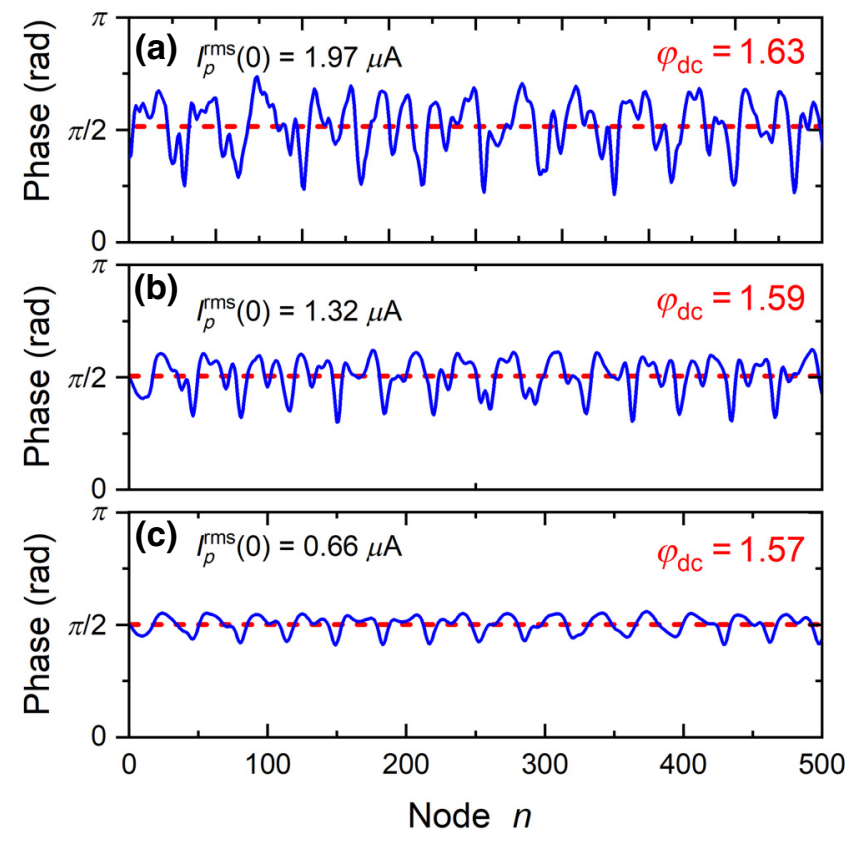

FIG. 4. Plots of WRSPICE junction phase between nodes 0 and 500 , taken at $t=15 \mathrm{~ns}$. (a) $I_{p}^{\mathrm{rms}}(0) \approx 1.97 \mu \mathrm{A}$, as per Ref. [16]. The phase is set to $\varphi_{\mathrm{dc}}=\pi / 2$. The strong pump current causes a large phase swing $(\approx \pm \pi / 4)$, and the dc bias moves away from the optimal position. (b) Pump current reduced to $I_{p}^{\mathrm{rms}}(0) \approx 1.40 \mu \mathrm{A}$. Both the phase swing and the dc offset are reduced. (c) Pump current used in simulations to investigate the JTWPA signal gain $I_{p}^{\mathrm{rms}}(0) \approx 0.67 \mu \mathrm{A}$. A minimal phase swing is observed, with the dc-bias position remaining at the optimal position $\left(\varphi_{\mathrm{dc}}=\pi / 2\right)$.

high pump currents, $I_{p}^{\mathrm{rms}}(0) \approx 2 \mu \mathrm{A}, \varphi_{\mathrm{ac}}$ can no longer be considered to be small in comparison with $\varphi_{\mathrm{dc}}$. In addition, rectification generated from the tones is absent in the CMEs. A consequence of significant rectification is a deviation from the optimal $\varphi_{\mathrm{dc}}=\pi / 2$ bias point such that the device no longer operates in the purely $3 \mathrm{WM}$ regime.

We extend the CMEs up to CME-5; this ensures that all of the tones remain below the cutoff frequency of our line, $\omega_{c}^{\prime} / 2 \pi=72.3 \mathrm{GHz}$. For such high input pump currents as those shown in Fig. 2, for which $I_{p}^{\mathrm{rms}}(0) \approx 1.97 \mu \mathrm{A}$, pump harmonic generation up to $f_{7 p}$ is observed (not shown in the figure); however, these tones cannot propagate over a significant number of cells of the amplifier, but they can be generated at each node. As we extend the CMEs only to $\mathrm{CME}-5$, we choose to reduce the input pump current such that pump harmonics beyond $f_{5 p}$ are insignificant, and that the assumption that $\varphi_{\mathrm{ac}}$ is small compared with $\varphi_{\mathrm{dc}}$ is upheld. Figure 4 shows that reducing the pump current from $I_{p}^{\mathrm{rms}}(0) \approx 1.97 \mu \mathrm{A}$ to $I_{p}^{\mathrm{rms}}(0) \approx 0.67 \mu$ A reduces the amplitude of $\varphi_{\mathrm{ac}}$ and better maintains a bias point of $\varphi_{\mathrm{dc}} \approx 1.57 \approx \pi / 2$. We also reduce the input signal to $I_{s}^{\mathrm{rms}}(0) \approx 0.05 \mu \mathrm{A}$. Note as we reduce both input amplitudes, CME-1 predicts a maximum signal gain at node
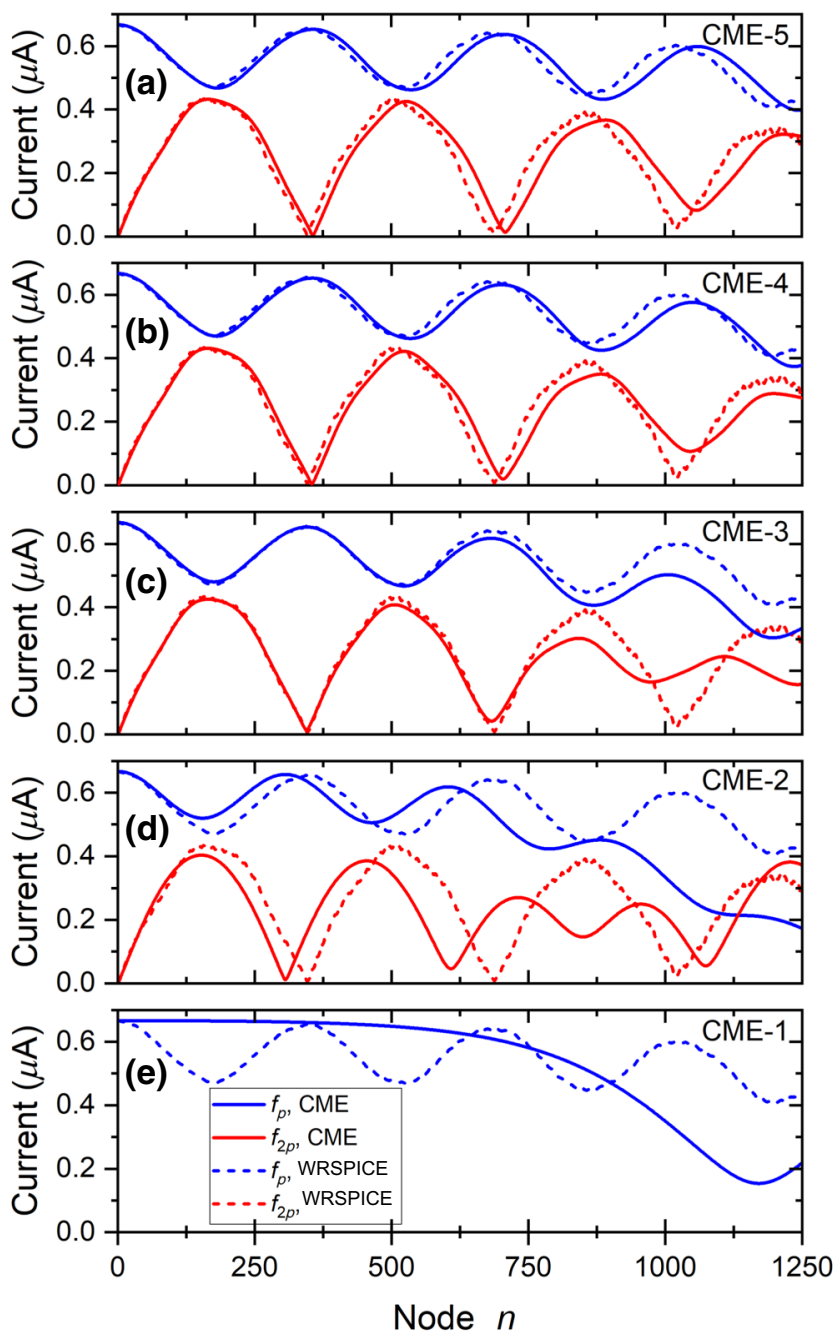

FIG. 5. Pump current and second harmonic of the pump current as a function of node number. $I_{p}^{\mathrm{rms}}(0) \approx 0.67 \mu \mathrm{A}\left[I_{s}^{\mathrm{rms}}(0) \approx\right.$ $0.05 \mu \mathrm{A}$ - signal trace not shown]. The WRSPICE simulations of the pump and second harmonic of the pump are shown by dashed lines, and are the same for each part of the figure. The CME$K$ solutions are shown by solid lines. Each part of the figure shows a different extension of CME. The agreement between the WRSPICE simulations and the CMEs reduces as the number of tones included in the CME is reduced. (a) CME-5, (b) CME-4, (c) CME-3, (d) CME-2, (e) CME-1.

$n \approx 1175$; therefore, we now consider an amplifier with 1250 nodes.

Figure 5 shows the current of the pump and the current of the second harmonic of the pump along the JTWPA. The fit of CME- 5 to the WRSPICE data is greatly improved when the pump current is at the lower amplitude of $I_{p}^{\mathrm{rms}}(0) \approx$ $0.67 \mu \mathrm{A}$, and the results remain in agreement over more nodes. Figure 5 also shows that reducing the number of allowed states in the set of equations (i.e., CME-5 $\rightarrow$ CME-4 $\rightarrow$ CME-3 $\rightarrow$ CME-2 $\rightarrow$ CME-1) results in an increased deviation between the CME solutions and the 
(a)

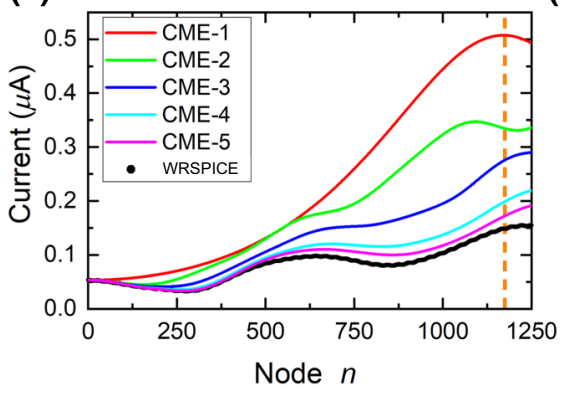

(b)

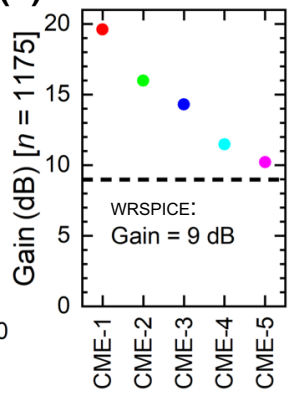

FIG. 6. (a) Signal current at each node of the JTWPA circuit in the WRSPICE simulations and for each CME extension. With increasing CME extension, we see improved agreement between the CME theory and the WRSPICE simulations. The traditional analytical theory, CME-1, predicts a maximum signal at node $n=1175$, corresponding to a gain of $20 \mathrm{~dB}$. We calculate the JTWPA gain for each CME set from the current measured at this node. (b) Gain measured at $n=1175$ for each CME extension. The WRSPICE simulation result is shown by a horizontal dashed line $(G=9 \mathrm{~dB})$. The measured gain for each CME set is reduced as the number of equations in the CME set increases. The gain measured approaches the value calculated from the WRSPICE simulations.

WRSPICE output. These results demonstrate the risk of reducing the number of tones represented in the CME set. As the number of tones is reduced, the behavior of the pump and the second harmonic of the pump is less well described. Indeed, Fig. 5(e) shows that if second-harmonic generation is neglected, as in CME-1, even qualitative comparability with the WRSPICE simulation is absent.

\section{Effect on signal gain}

Figure 6(a) shows the signal current at each node of the JTWPA in the WRSPICE simulations and for CME-1 to CME-5. It can be seen that the presence of additional tones in the CMEs leads to a reduction in gain. CME-5 and WRSPICE are in fair agreement and exhibit the least gain.

To quantify the reduction in gain observed as the CMEs are extended, we choose the optimal-gain node of CME$1(n=1175)$ and compare the gain at this node with the results from the other CMEs and the WRSPICE simulation at this node. Figure 6(b) shows that as the number of terms in the CMEs increase, we capture more complex behavior of the signal as well as the detrimental effect on the gain. WRSPICE includes all tones propagating along the JTWPA, as noted earlier, and shows an even lower gain than does CME-5 at node $n=1175$.

Figure 6(a) also shows deamplification of the signal at the beginning of the JTWPA up to approximately node 300 . We believe that this deamplification is due to the signal current dispersing into other mixing tones. All tones, with the exception of the pump and the signal, are input into the equations with zero initial amplitude, and thus the current required to generate these tones must initially come from the pump and signal. It is observed that as the number of tones included in the CMEs increases, the number of nodes over which the signal is deamplified increases, although the initial gradient is unchanged.

\section{DISCUSSION AND CONCLUSION}

Our extension of the CMEs shows that CME-1 (including only the pump, signal, and idler) is insufficient to capture the complex behavior of the JTWPA. As we increase the number of terms in the CMEs, we approach the behavior and gain figures observed in the WRSPICE simulations. We note that whilst good agreement between CME-5 and WRSPICE is achieved, there is still not full agreement. We now comment on the possible sources of the remaining discrepancy.

Only the quadratic term in the current-phase relation of the flux-biased SQUID is included in the formation of the CMEs. Inclusion of the quartic (and higher-order) terms might bring the WRSPICE and CME results into even better agreement. The dc offset generated by all other tones (analogous to optical rectification) is also not included in the CMEs, whilst a dc current contribution is seen in the WRSPICE results for high pump currents. Finally, our choice of CME extensions is based on the WRSPICE results, which show large-amplitude pump-harmonic and pumpmediated tones. Only these tones are included in the CME extensions that we present in this paper. Additional tones, including higher harmonics of the signal, may need consideration for improved agreement between WRSPICE and the CMEs (some of these tones are apparent in the WRSPICE simulations and are discussed in Appendix D).

We believe that these results will have practical consequences for the design and operation of JTWPAs, in particular for considerations of measurement bandwidth, tone reflections, and optimization procedures.

To conclude, we demonstrate that a simple consideration of only three tones is insufficient to describe the complex behavior of the JTWPA. We present four further extensions of the coupled mode equations, increasing the number of interacting tones included with each extension. We also use WRSPICE to simulate the JTWPA, and compare its output with that of the extended coupled mode equations. Each further extension of the CMEs agrees more accurately with the WRSPICE simulation.

In order to design an amplifier and to obtain representative gain figures, the full behavior of the JTWPA should be included. The CMEs do not represent the full behavior of the JTWPA line - for instance, reflections are not included, and deviation from the purely $3 \mathrm{WM}$ working point is not considered. In addition, it is nontrivial to include stop-band engineering in the CME description. Use of a circuit solver enables testing of modified 
JTWPA designs (including dispersion engineering) without the need to derive CMEs and a dispersion relation from first principles for each design iteration. In this regard, a circuit solver such as WRSPICE, which includes the effects of these physical features and allows JTWPA designs to be more fully simulated and to more accurately represent the physical device, is superior. Both the simulations and the extended CME analytical theory show clearly that the generation of pump harmonics and the pump-mediated sum-frequency generation terms must be considered when designing such a broadband device. In order to achieve the gain required for a usable quantum-limited amplifier, engineering to suppress the generation of pump harmonics may need to be implemented. Some of this engineering has already been considered in the form of stop-band engineering $[14,16,20,21]$.

The work presented here realizes a simple computationally inexpensive method for extension of the CMEs to describe propagators that have been previously neglected and demonstrates the utility of WRSPICE for the simulation of nonlinear superconducting circuits, in particular as a design tool for JTWPAs.

\section{ACKNOWLEDGMENTS}

This project received funding from the EMPIR program, cofinanced by the Participating States, and from the European Union's Horizon 2020 research and innovation program. This work is part of the joint research project PARAWAVE, and we would like to thank members of the consortium, in particular R. Dolata, M. Khabipov, C. Kißling, and A. B. Zorin, for useful discussions on the operation of the JTWPA. The work is partially supported by the UK Department of Business, Energy and Industrial Strategy (BEIS). We thank J. J. Burnett and J. C. Gallop for critical review of the manuscript.

\section{APPENDIX A: WRSPICE IMPLEMENTATION OF JTWPA}

The WRSPICE implementation of the JTWPA is shown in Fig. 7. The blue dashed lines show the repeating rf-SQUID cell. The rf SQUID contains a Josephson junction, represented by a nonlinear inductance $L_{J}$ and a capacitance $C_{J}$, and a geometric inductance, represented by $L_{g}$. The de flux in the rf SQUID is set by a dc current source $I_{\mathrm{dc}}$ and a coupled inductor $L_{\text {flux }}$. Each cell has a capacitance to ground $C_{0}$. The cells can be repeated as many times as required. The JTWPA described in the main text has 1250 cells. The pump $\left(I_{p}\right)$ and signal $\left(I_{S}\right)$ tones are input into the front of the JTWPA. The JTWPA is terminated with a resistance $R_{\text {term }}$. The parameters of the circuit presented in this paper are $I_{c}=5 \mu \mathrm{A}, C_{J}=60 \mathrm{fF}, C_{0}=100 \mathrm{fF}, L_{g}=57 \mathrm{pH}$, and $R_{\text {term }}=Z_{0}=23.87 \Omega$. The shunt resistance of the junction is set to infinity in the WRSPICE junction model used in the

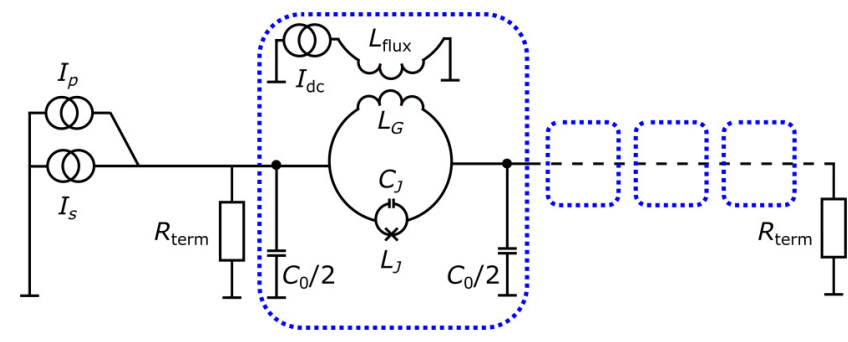

FIG. 7. Circuit schematic of the JTWPA as implemented in WRSPICE. The rf-SQUID cell, including the dc-flux-bias circuit, is highlighted by the blue dashed lines. The cells in our circuit are repeated $N$ times (dependent on the length of the amplifier). The pump and signal are input at the front of the JTWPA, and the line is terminated with a termination resistor. The parameters of the circuit presented in this paper are $I_{c}=5 \mu \mathrm{A}, C_{J}=$ $60 \mathrm{fF}, C_{0}=100 \mathrm{fF}, L_{g}=57 \mathrm{pH}$, and $R_{\text {term }}=23.87 \Omega$. The full WRSPICE circuit is shown in Ref. [22].

present work. The full WRSPICE circuit file for our JTWPA implementation contains all circuit parameters used [22].

\section{APPENDIX B: JTWPA BANDWIDTH}

A key figure of merit of an amplifier is its gain-versusfrequency characteristic. Figure 8 shows the gain versus frequency for the JTWPA for each CME extension and the WRSPICE simulation. It is observed that as the CME set is extended, the gain reduces over the frequency range investigated $(2-10 \mathrm{GHz})$. In addition, the gain-versusfrequency characteristic simulated by WRSPICE shows a further decrease across the range. The closest agreement to the WRSPICE simulation is again provided by the results of CME-5.

\section{APPENDIX C: TRANSMISSION-LINE THEORY}

An ideal simple continuous series- $L$ and parallel- $C$ transmission line, with the inductance and capacitance expressed as $L^{\prime}$ and $C^{\prime}$ per unit length, respectively, allows all electromagnetic waves to propagate with the same velocity, given by

$$
v_{1}=\frac{\omega}{k_{1}}=\frac{1}{\sqrt{L^{\prime} C^{\prime}}}
$$

where $k_{1}=2 \pi / \lambda_{1}$ and $\omega=2 \pi f$. Such a line does not have a physical cutoff frequency. By contrast, a lumpedelement $L-C$ transmission line representing a Josephson traveling-wave parametric amplifier, illustrated in Fig. 9, has a propagation constant $F=I_{B C} / I_{A B}$, and so on throughout the line [23]. This constant can be written as $F=e^{-\Gamma}$, where $\Gamma$ is a complex number equal to $\alpha+i \theta$. Such a line does have a physical cutoff frequency, $\omega_{c}=2 / \sqrt{L_{g} C_{0}}$, below which electromagnetic waves are propagated without attenuation, and the constant $\alpha$ is zero. 


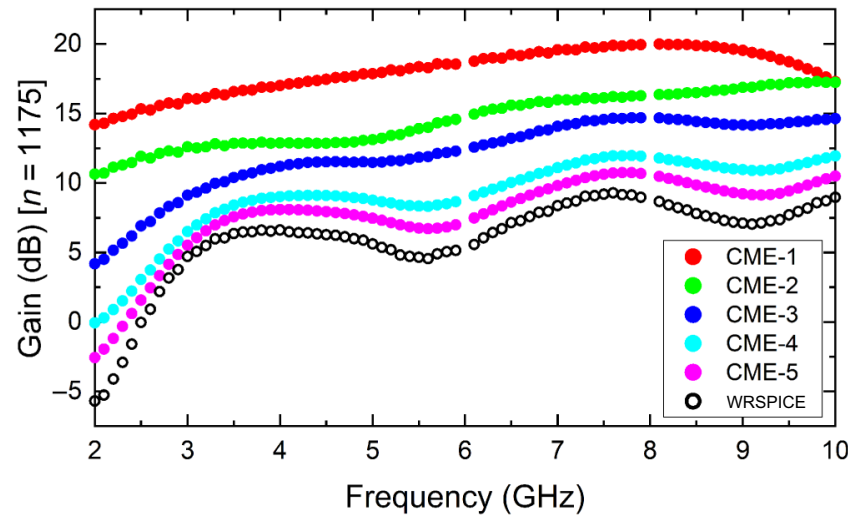

FIG. 8. Gain versus signal frequency for the JTWPA for each CME- $K$ compared with the WRSPICE output (at a fixed pump frequency $f_{p}=12 \mathrm{GHz}$ ). As the CME is extended, the gain broadly reduces over the frequency range investigated. The WRSPICE gain-versus-frequency curve agrees best with $\mathrm{CME}-5$, the furthest $\mathrm{CME}$ extension considered in this paper. Note that the degenerate case $\left(f_{s}=6 \mathrm{GHz}\right)$ is not shown. Also omitted is the case where $f_{s}=8 \mathrm{GHz}$ - this is because the second harmonic of the idler then occurs at the signal frequency $\left(2 f_{i}=f_{s}\right)$, which is not considered in our CMEs.

In this frequency range, the phase shift per cell is given by

$$
\cos \theta=1-\frac{\omega^{2} L_{g} C_{0}}{2}=1-\frac{2 \omega^{2}}{\omega_{c}^{2}}
$$

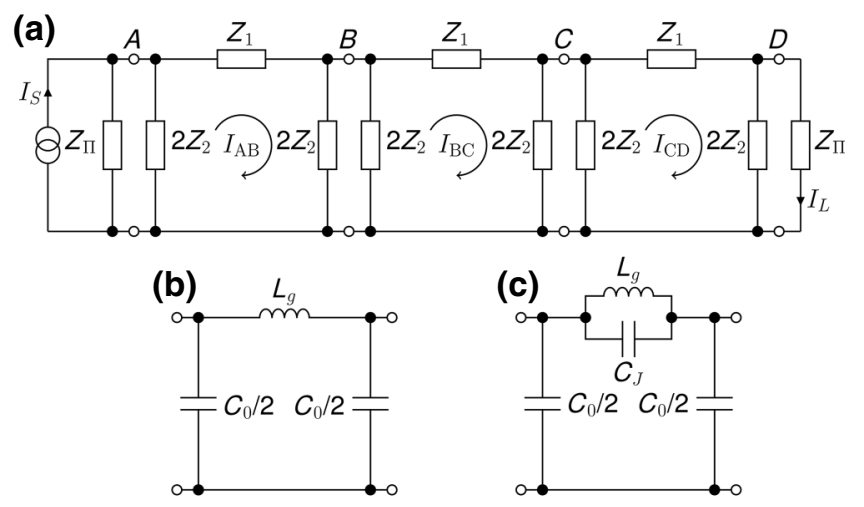

FIG. 9. (a) Ladder network of $\Pi$ sections. Each section of the transmission line has a series impedance $Z_{1}$ and a parallel impedance $Z_{2}$. We consider two versions of the transmission-line sections: (b) a lumped-element $L-C$ section, where $Z_{1}$ represents the impedance of the series inductance $L_{g}\left(Z_{1}=i \omega L_{g}\right)$ and $Z_{2}$ represents the impedance of the parallel capacitance $C_{0}$ $\left(Z_{2}=1 / i \omega C_{0}\right)$; (c) a lumped-element $L-C$ section with a modification to $Z_{1}$ to include the junction capacitance $C_{J}$ in parallel with the inductor $L_{g}$, such that $Z_{1}=i \omega L_{g} /\left(1-\omega^{2} L_{g} C_{J}\right)$.
If the physical length of the sections is $a$, then the velocity of the wave along the line can be deduced to be

$$
v_{2}=\frac{\omega}{k_{2}}=\frac{\omega a}{\theta}=\frac{\omega a}{\arccos \left(1-2 \omega^{2} / \omega_{c}^{2}\right)},
$$

where $k_{2}=2 \pi / \lambda_{2}$. If the cosine is approximated as $\cos \theta \approx 1-\theta^{2} / 2$, then $\theta \approx \omega \sqrt{L_{g} C_{0}}$, from which it can be deduced that the time delay per section is $\Delta t \approx \sqrt{L_{g} C_{0}}$. The velocity of the wave along the line for frequencies well below the cutoff frequency simplifies to $v_{2} \approx a / \sqrt{L_{g} C_{0}}$, which tends to the same result as that for a continuous transmission line with $L^{\prime}=L_{g} / a$ and $C^{\prime}=C_{0} / a$. However, as the cutoff frequency is approached, the wave velocity in a lumped line deviates from a constant value, with the phase shift per cell being described by Eq. (C2). At $\omega=$ $\omega_{c}$, the wavelength becomes equal to $2 a$, corresponding to $\theta=\pi$.

If the lumped-element line is now modified to include a capacitance $C_{J}$ in parallel with the inductor $L_{g}$ to represent the capacitance of the Josephson junction in the SQUID element, then the cutoff frequency becomes

$$
\omega_{c}^{\prime}=\frac{2}{\sqrt{L_{g}\left(C_{0}+4 C_{J}\right)}},
$$

and the relation for $\theta$ is

$$
\cos \theta=1-\frac{\omega^{2} L_{g} C_{0}}{2\left(1-\omega^{2} L_{g} C_{J}\right)}=1-\frac{2 \omega^{2}}{\omega_{c}^{2}\left(1-\omega^{2} / \omega_{J}^{2}\right)} .
$$

If the small-angle approximation for $\cos \theta$ is again used, then Eq. (C5) becomes

$$
\theta \approx \frac{2 \omega}{\omega_{c} \sqrt{\left(1-\omega^{2} / \omega_{J}^{2}\right)}}=k_{2}^{\prime} a .
$$

This equation compares exactly with Eq. (6), with the substitution $\omega_{c}=2 a / \sqrt{L_{g} C_{0}}$. For our CME solution, we adopt the expression for $\cos \theta$ to obtain

$$
k=\frac{1}{a} \arccos \left(1-\frac{2 \omega^{2}}{\omega_{c}^{2}\left(1-\omega^{2} / \omega_{J}^{2}\right)}\right) .
$$

The functions described by Eqs. (C6) and (C7) and the data obtained from WRSPICE are plotted in Fig. 10. The phase change per cell of the lumped-element line (black dots, WRSPICE output) is described by $k$ [blue line, Eq. (6)]. The small-angle approximation used to obtain $\theta \approx k_{2}^{\prime} a$ [Eq. (C6), red line] only describes the lumped-element line adequately at lower frequencies, and deviates from the WRSPICE output beyond approximately $40 \mathrm{GHz}$. For all CME- $K$ simulations presented in the main text, the exact form [Eq. (C7)] is used. 


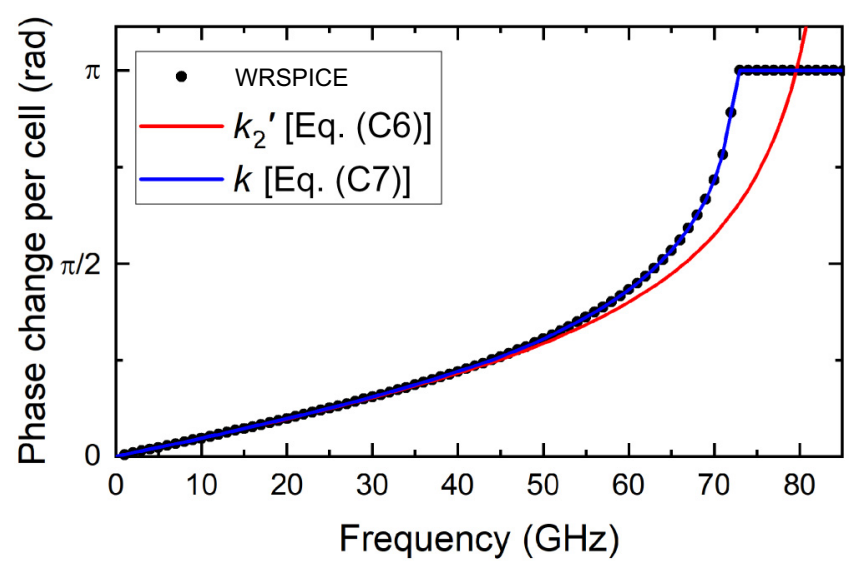

FIG. 10. Phase shift per cell as a function of the electromagnetic-wave frequency. The red line shows the relation for $k_{2}^{\prime}$ (with $a$ set to 1) described by Eq. (C6) this compares exactly to the dispersion for a continuous line as described by Eq. (6). The blue line shows the relation for $k$ (with $a$ set to 1) described by Eq. (C7), which is used in our CME solutions. The phase shift per cell is also obtained from WRSPICE simulations of the transmission line shown in Fig. 9(a) with the cell shown in Fig. 9(c).

Figure 11 shows the effect of using the exact phase change per cell described by Eq. (C7) and of using the approximation given by Eq. (C6). The exact expression agrees with the WRSPICE output better than the approximation does. The amplitude remains in line with that of the WRSPICE output over a higher number of nodes.

We note that the CME-5 solutions still deviate from the WRSPICE simulations as the distance along the amplifier is increased. One possibility is the lack of inclusion of reflections in the CME solutions. The ideal termination impedance at each end of the transmission line in Fig. 9 is

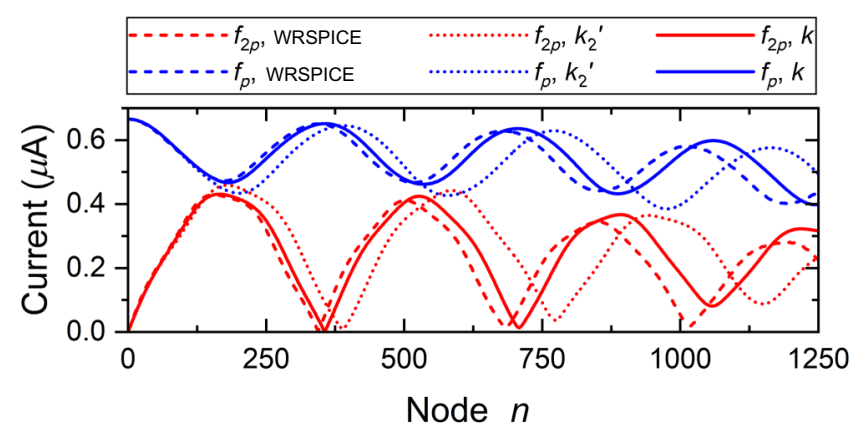

FIG. 11. Comparison of WRSPICE simulations (dashed lines) and the solutions to CME-5 $\left(f_{p}\right.$ and $\left.f_{2 p}\right)$ for both the exact phase-change-per-cell expression used throughout the main text [Eq. (C7), solid lines] and the approximation [Eq. (C6), dotted lines]. The solutions to CME-5 are in greater agreement with the WRSPICE simulations when the exact phase-change-per-cell expression is used.

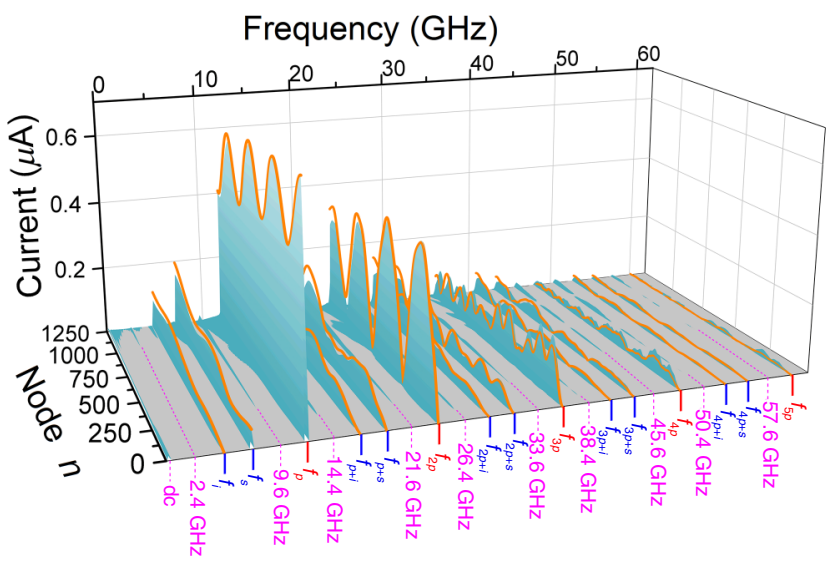

FIG. 12. Output of WRSPICE (surface plot) shown in combination with the corresponding CME-5 solutions (orange lines). The pump-harmonic tones are labeled in red, and the pumpmediated mixing products in blue. The CME-5 solutions are in agreement with the WRSPICE output for all tones considered. We note also that other tones not considered in CME-5 are present in the WRSPICE output and are labeled by frequency in magenta. Note that the gray surface is set to $3 \mathrm{nA}$ to highlight clearly the emergent tones not considered in CME-5.

given by

$$
Z_{\Pi}=\sqrt{\frac{L_{g}}{C_{0}\left(1-\omega^{2} / \omega_{c}^{\prime 2}\right)}} .
$$

In our WRSPICE simulations, we use a fixed value of $Z_{0}=$ $\sqrt{L_{g} / C_{0}}$ for the termination impedances to represent the practical situation. However, it can be seen that $Z_{\Pi}$ deviates from $Z_{0}$ as $\omega$ reaches $\omega_{c}$, eventually reaching infinity when $\omega=\omega_{c}$. There will therefore be reflected voltages and currents on the transmission line in both a practical amplifier and the WRSPICE simulation, which are not accounted for in the CME solution.

\section{APPENDIX D: FULL WRSPICE OUTPUT, INCLUDING COMPARISON WITH ALL TONES IN CME-5}

In the main text, we focus on the agreement between CME-5 and the tones of major interest - the pump, the second harmonic of the pump, and the signal. Figure 12 shows the WRSPICE output (blue surfaces) and the CME-5 solutions (orange lines) for all tones shown in Table I. Each tone included in the CME-5 solutions has a clear counterpart in the WRSPICE output, and there is agreement between the two in the amplitude at each node. The agreement of the fit reduces toward the output end of the amplifier.

The WRSPICE output provides a full spectrum of frequencies for the JTWPA and clearly shows additional emergent tones not included in CME-5. A dc current is 


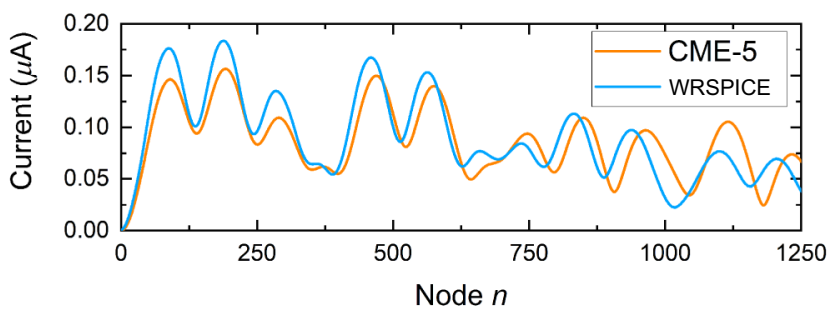

FIG. 13. WRSPICE and CME-5 solutions for the 36-GHz tone. The two methods are in agreement over the useful length of the amplifier. Notably, the CME-5 solutions underpredict the tone amplitude as simulated in WRSPICE. This is likely due to interactions that can produce $36 \mathrm{GHz}$ that are not considered in CME-5 but can be produced in WRSPICE - for instance, generation of the third harmonic of the pump.

present, which is attributed to the aforementioned rectification. There are also tones present at 2.4, 9.6, and 14.4 $\mathrm{GHz}$ and so on.

The 2.4-GHz tone is generated via difference-frequency generation (DFG) from the signal and the idler $\left(f_{s}-f_{i}=\right.$ $f_{s-i}=2.4 \mathrm{GHz}$ ) and is a tone used in later interactions. Each tone can be generated via multiple different interaction routes. As a brief example, we show how the 14.4$\mathrm{GHz}$ tone can be produced through different interaction routes; second-harmonic generation from the input signal tone $\left(f_{s}+f_{s}=14.4 \mathrm{GHz}\right)$, DFG from $f_{p+s}$ and $f_{i}\left(f_{p+s}-\right.$ $f_{i}=14.4 \mathrm{GHz}$ ), and sum-frequency generation (SFG) from $f_{p}$ and $f_{s-i}\left(f_{p}+f_{s-i}=14.4 \mathrm{GHz}\right)$. It is also possible to generate this tone by SFG from $f_{2 i}$ and $f_{i}\left(f_{2 i}+f_{i}=14.4 \mathrm{GHz}\right)$. As stated in the main text, the CMEs consider only pumpmediated and pump-harmonic-mediated tones. The above mechanisms to create $14.4 \mathrm{GHz}$ demonstrate that the tones can be generated in a myriad of ways, including ways that do not include the pump tone or a pump-harmonic tone. These mixing mechanisms are not included in the CMEs but are manifested in WRSPICE, and may be present in a real amplifier, resulting in the generation or interaction of additional tones at various frequencies.

Finally, we point out another possible mechanism not considered in the CMEs - tone generation via 4WM. Figure 4 shows that the junction phase deviates from $\varphi_{\mathrm{dc}}=$ $\pi / 2$, that is, both $3 \mathrm{WM}$ and $4 \mathrm{WM}$ will occur. An example of this is generation of the third harmonic of the idler $\left(f_{i}+f_{i}+f_{i}=14.4 \mathrm{GHz}\right)$ to again produce the $14.4-\mathrm{GHz}$ tone.

Figure 13 shows the WRSPICE output and CME-5 solution for the $36-\mathrm{GHz}$ tone. This tone is notable because, for a large range of the simulation, the CME- 5 solution underpredicts the WRSPICE output. For the majority of tones considered in CME-5, the reverse is true. Again, a potential explanation for this deviation between WRSPICE and CME5 is the presence of $4 \mathrm{WM}$. WRSPICE allows $4 \mathrm{WM}$ to occur, whilst the CME-5 equations have no possibility of 4WM. Generation of the third harmonic of the pump (12 GHz) creates a tone at $36 \mathrm{GHz}$. It is possible that this $4 \mathrm{WM}$ route to generation of the $36-\mathrm{GHz}$ tone explains some of the deviation in the agreement between WRSPICE and CME-5.

\section{APPENDIX E: EXTENSION TO CME-3}

CME- 3 extends CME- 2 by including the third harmonic of the pump $f_{3 p}$, and the sum-frequency terms $f_{2 p+i}$ and $f_{2 p+s}$. We show in detail all of the terms included in the coupled mode equations forming CME-3:

$$
\begin{aligned}
\frac{d A_{p}}{d x}= & \frac{\beta}{2}\left(-k_{s} k_{i} A_{s} A_{i} e^{i\left(k_{s}+k_{i}\right) x}+k_{2 p} A_{2 p} k_{p} A_{p}^{*} e^{i\left(k_{2 p}-k_{p}\right)}+k_{p+s} k_{s} A_{p+s} A_{s}^{*} e^{i\left(k_{p+s}-k_{s}\right) x}\right. \\
& +k_{p+i} k_{i} A_{p+i} A_{i}^{*} e^{i\left(k_{p+i}-k_{i}\right) x}+k_{3 p} k_{2 p} A_{3 p} A_{2 p}^{*} e^{i\left(k_{3 p}-k_{2 p}\right) x} \\
& \left.+k_{2 p+s} k_{p+s} A_{2 p+s} A_{p+s}^{*} e^{i\left(k_{2 p+s}-k_{p+s}\right) x}+k_{2 p+i} k_{p+i} A_{2 p+i} A_{p+i}^{*} e^{i\left(k_{2 p+i}-k_{p+i}\right) x}\right) e^{-i k_{p} x}, \\
\frac{d A_{s}}{d x}= & \frac{\beta}{2}\left(k_{i} k_{p} A_{i}^{*} A_{p} e^{i\left(k_{p}-k_{i}\right) x}+k_{p} k_{p+s} A_{p}^{*} A_{p+s} e^{i\left(k_{p+s}-k_{p}\right) x}+k_{2 p} k_{p+i} A_{p+i}^{*} A_{2 p} e^{i\left(k_{2 p}-k_{p+i}\right) x}\right. \\
& \left.+k_{3 p} k_{2 p+i} A_{3 p} A_{2 p+i}^{*} e^{i\left(k_{3 p}-k_{2 p+i}\right) x}+k_{2 p+s} k_{2 p} A_{2 p+s} A_{2 p}^{*} e^{i\left(k_{2 p+s}-k_{2 p}\right) x}\right) e^{-i k_{s} x}, \\
\frac{d A_{i}}{d x}= & \frac{\beta}{2}\left(k_{s} k_{p} A_{s}^{*} A_{p} e^{i\left(k_{p}-k_{s}\right) x}+k_{p} k_{p+i} A_{p}^{*} A_{p+i} e^{i\left(k_{p+i}-k_{p}\right) x}+k_{2 p} k_{p+s} A_{p+s}^{*} A_{2 p} e^{i\left(k_{2 p}-k_{p+s}\right) x}\right. \\
& \left.+k_{3 p} k_{2 p+s} A_{3 p} A_{2 p+s}^{*} e^{i\left(k_{3 p}-k_{2 p+s}\right) x}+k_{2 p+i} k_{2 p} A_{2 p+i} A_{2 p}^{*} e^{i\left(k_{2 p+i}-k_{2 p}\right) x}\right) e^{-i k_{i} x}, \\
\frac{d A_{2 p}}{d x}= & \frac{\beta}{4}\left(k_{p}^{2} A_{p}^{2} e^{i\left(2 k_{p}\right) x}-k_{i} k_{p+s} A_{i} A_{p+s} e^{i\left(k_{i}+k_{p+s}\right) x}-k_{p+i} k_{s} A_{p+i} A_{s} e^{i\left(k_{p+i}+k_{s}\right) x}\right. \\
& \left.+k_{p} k_{3 p} A_{p}^{*} A_{3 p} e^{i\left(k_{3 p}-k_{p}\right) x}+k_{2 p+s} k_{s} A_{2 p+s} A_{s}^{*} e^{i\left(k_{2 p+s}-k_{s}\right) x}+k_{2 p+i} k_{i} A_{2 p+i} A_{i}^{*} e^{i\left(k_{2 p+i}-k_{i}\right) x}\right) e^{-i k_{2 p} x},
\end{aligned}
$$




$$
\begin{aligned}
\frac{d A_{p+s}}{d x}= & \frac{\beta}{2}\left(-k_{p} k_{s} A_{p} A_{s} e^{i\left(k_{p}+k_{s}\right) x}+k_{i} k_{2 p} A_{i}^{*} A_{2 p} e^{i\left(k_{2 p}-k_{i}\right) x}\right. \\
& \left.+k_{3 p} k_{p+i} A_{3 p} A_{p+i}^{*} e^{i\left(k_{3 p}-k_{p+i}\right) x}+k_{2 p+s} k_{p} A_{2 p+s} A_{p}^{*} e^{i\left(k_{2 p+s}-k_{p}\right) x}\right) e^{-i k_{p+s} x}, \\
\frac{d A_{p+i}}{d x}= & \frac{\beta}{2}\left(-k_{p} k_{i} A_{p} A_{i} e^{i\left(k_{p}+k_{i}\right) x}+k_{s} k_{2 p} A_{s}^{*} A_{2 p} e^{i\left(k_{2 p}-k_{s}\right) x}\right. \\
& \left.+k_{3 p} k_{p+s} A_{3 p} A_{p+s}^{*} e^{i\left(k_{3 p}-k_{p+s}\right) x}+k_{2 p+i} k_{p} A_{2 p+i} A_{p}^{*} e^{i\left(k_{2 p+i}-k_{p}\right) x}\right) e^{-i k_{p+i} x}, \\
\frac{d A_{3 p}}{d x}= & \frac{\beta}{2}\left(-k_{p} k_{2 p} A_{p} A_{2 p} e^{i\left(k_{p}+k_{2 p}\right) x}-k_{p+s} k_{p+i} A_{p+s} A_{p+i} e^{i\left(k_{p+s}+k_{p+i}\right) x}\right. \\
& \left.-k_{s} k_{2 p+i} A_{s} A_{2 p+i} e^{i\left(k_{s}+k_{2 p+i}\right) x}-k_{i} k_{2 p+s} A_{i} A_{2 p+s} e^{i\left(k_{i}+k_{2 p+s}\right) x}\right) e^{-i k_{3 p} x}, \\
\frac{d A_{2 p+s}}{d x}= & \frac{\beta}{2}\left(-k_{p} k_{p+s} A_{p} A_{p+s} e^{i\left(k_{p}+k_{p+s}\right) x}-k_{s} k_{2 p} A_{s} A_{2 p} e^{i\left(k_{s}+k_{2 p}\right) x}+k_{3 p} k_{i} A_{3 p} A_{i}^{*} e^{i\left(k_{3 p}-k_{i}\right) x}\right) e^{-i k_{2 p+s} x}, \\
\frac{d A_{2 p+i}}{d x}= & \frac{\beta}{2}\left(-k_{p} k_{p+i} A_{p} A_{p+i} e^{i\left(k_{p}+k_{p+i}\right) x}-k_{i} k_{2 p} A_{i} A_{2 p} e^{i\left(k_{i}+k_{2 p}\right) x}+k_{3 p} k_{s} A_{3 p} A_{s}^{*} e^{i\left(k_{3 p}-k_{s}\right) x}\right) e^{-i k_{2 p+i} x} .
\end{aligned}
$$

\section{APPENDIX F: EXTENSION TO CME-4}

The penultimate coupled-mode-equation extension that we present in full is the extension from CME-3 to CME-4 by inclusion of the fourth harmonic of the pump $f_{4 p}$, and the sum-frequency terms $f_{3 p+i}$ and $f_{3 p+s}$. The full list of tones included in CME-4 is shown in Table I. We show below in detail all of the terms included in the coupled mode equations forming CME-4:

$$
\begin{aligned}
\frac{d A_{p}}{d x}= & \frac{\beta}{2}\left(-k_{s} k_{i} A_{s} A_{i} e^{i\left(k_{s}+k_{i}\right) x}+k_{2 p} A_{2 p} k_{p} A_{p}^{*} e^{i\left(k_{2 p}-k_{p}\right) x}+k_{p+s} k_{s} A_{p+s} A_{s}^{*} e^{i\left(k_{p+s}-k_{s}\right) x}\right. \\
& +k_{p+i} k_{i} A_{p+i} A_{i}^{*} e^{i\left(k_{p+i}-k_{i}\right) x}+k_{3 p} k_{2 p} A_{3 p} A_{2 p}^{*} e^{i\left(k_{3 p}-k_{2 p}\right) x} \\
& +k_{2 p+s} k_{p+s} A_{2 p+s} A_{p+s}^{*} e^{i\left(k_{2 p+s}-k_{p+s}\right) x}+k_{2 p+i} k_{p+i} A_{2 p+i} A_{p+i}^{*} e^{i\left(k_{2 p+i}-k_{p+i}\right) x} \\
& +k_{4 p} k_{3 p} A_{4 p} A_{3 p}^{*} e^{i\left(k_{4 p}-k_{3 p}\right) x}+k_{3 p+s} k_{2 p+s} A_{3 p+s} A_{2 p+s}^{*} e^{i\left(k_{3 p+s}-k_{2 p+s}\right) x} \\
& \left.+k_{3 p+i} k_{2 p+i} A_{3 p+i} A_{2 p+i}^{*} e^{i\left(k_{3 p+i}-k_{2 p+i}\right) x}\right) e^{-i k_{p} x}, \\
\frac{d A_{s}}{d x}= & \frac{\beta}{2}\left(k_{i} k_{p} A_{i}^{*} A_{p} e^{i\left(k_{p}-k_{i}\right) x}+k_{p} k_{p+s} A_{p}^{*} A_{p+s} e^{i\left(k_{p+s}-k_{p}\right) x}+k_{2 p} k_{p+i} A_{p+i}^{*} A_{2 p} e^{i\left(k_{2 p}-k_{p+i}\right) x}\right. \\
& +k_{3 p} k_{2 p+i} A_{3 p} A_{2 p+i}^{*} e^{i\left(k_{3 p}-k_{2 p+i}\right) x}+k_{2 p+s} k_{2 p} A_{2 p+s} A_{2 p}^{*} e^{i\left(k_{2 p+s}-k_{2 p}\right) x} \\
& \left.+k_{4 p} k_{3 p+i} A_{4 p} A_{3 p+i}^{*} e^{i\left(k_{4 p}-k_{3 p+i}\right) x}+k_{3 p} k_{3 p+s} A_{3 p+s} A_{3 p}^{*} e^{i\left(k_{3 p+s}-k_{3 p}\right) x}\right) e^{-i k_{s} x}, \\
\frac{d A_{i}}{d x}= & \frac{\beta}{2}\left(k_{s} k_{p} A_{s}^{*} A_{p} e^{i\left(k_{p}-k_{s}\right) x}+k_{p} k_{p+i} A_{p}^{*} A_{p+i} e^{i\left(k_{p+i}-k_{p}\right) x}+k_{2 p} k_{p+s} A_{p+s}^{*} A_{2 p} e^{i\left(k_{2 p}-k_{p+s}\right) x}\right. \\
& +k_{3 p} k_{2 p+s} A_{3 p} A_{2 p+s}^{*} e^{i\left(k_{3 p}-k_{2 p+s}\right) x}+k_{2 p+i} k_{2 p} A_{2 p+i} A_{2 p}^{*} e^{i\left(k_{2 p+i}-k_{2 p}\right) x} \\
& \left.+k_{4 p} k_{3 p+s} A_{4 p} A_{3 p+s}^{*} e^{i\left(k_{4 p}-k_{3 p+s}\right) x}+k_{3 p} k_{3 p+i} A_{3 p+i} A_{3 p}^{*} e^{i\left(k_{3 p+i}-k_{3 p}\right) x}\right) e^{-i k_{i} x} \\
\frac{d A_{2 p}}{d x}= & \frac{\beta}{2}\left(-\frac{k_{p}^{2} A_{p}^{2}}{2} e^{i\left(k_{p}+k_{p}\right) x}-k_{i} k_{p+s} A_{i} A_{p+s} e^{i\left(k_{i}+k_{p+s}\right) x}-k_{p+i} k_{s} A_{p+i} A_{s} e^{i\left(k_{p+i}+k_{s}\right) x}\right. \\
& +k_{p} k_{3 p} A_{p}^{*} A_{3 p} e^{i\left(k_{3 p}-k_{p}\right) x}+k_{2 p+s} k_{s} A_{2 p+s} A_{s}^{*} e^{i\left(k_{2 p+s}-k_{s}\right) x}
\end{aligned}
$$




$$
\begin{aligned}
& +k_{2 p+i} k_{i} A_{2 p+i} A_{i}^{*} e^{i\left(k_{2 p+i}-k_{i}\right) x}+k_{4 p} k_{2 p+i} A_{4 p} A_{2 p}^{*} e^{i\left(k_{4 p}-k_{2 p}\right) x} \\
& \left.+k_{3 p+s} k_{p+s} A_{3 p+s} A_{p+s}^{*} e^{i\left(k_{3 p+s}-k_{p+s}\right) x}+k_{3 p+i} k_{p+i} A_{3 p+i} A_{p+i}^{*} e^{i\left(k_{3 p+i}-k_{p+i}\right) x}\right) e^{-i k_{2 p} x}, \\
& \frac{d A_{p+s}}{d x}=\frac{\beta}{2}\left(-k_{p} k_{s} A_{p} A_{s} e^{i\left(k_{p}+k_{s}\right) x}+k_{i} k_{2 p} A_{i}^{*} A_{2 p} e^{i\left(k_{2 p}-k_{i}\right) x}\right. \\
& +k_{3 p} k_{p+i} A_{3 p} A_{p+i}^{*} e^{i\left(k_{3 p}-k_{p+i}\right) x}+k_{2 p+s} k_{p} A_{2 p+s} A_{p}^{*} e^{i\left(k_{2 p+s}-k_{p}\right) x} \\
& \left.+k_{4 p} k_{2 p+i} A_{4 p} A_{2 p+i}^{*} e^{i\left(k_{4 p}-k_{2 p+i}\right) x}+k_{3 p+s} k_{2 p} A_{3 p+s} A_{2 p}^{*} e^{i\left(k_{3 p+s}-k_{2 p}\right) x}\right) e^{-i k_{p+s} x}, \\
& \frac{d A_{p+i}}{d x}=\frac{\beta}{2}\left(-k_{p} k_{i} A_{p} A_{i} e^{i\left(k_{p}+k_{i}\right) x}+k_{s} k_{2 p} A_{s}^{*} A_{2 p} e^{i\left(k_{2 p}-k_{s}\right) x}\right. \\
& +k_{3 p} k_{p+s} A_{3 p} A_{p+s}^{*} e^{i\left(k_{3 p}-k_{p+s}\right) x}+k_{2 p+i} k_{p} A_{2 p+i} A_{p}^{*} e^{i\left(k_{2 p+i}-k_{p}\right) x} \\
& \left.+k_{4 p} k_{2 p+s} A_{4 p} A_{2 p+s}^{*} e^{i\left(k_{4 p}-k_{2 p+s}\right) x}+k_{3 p+i} k_{2 p} A_{3 p+i} A_{2 p}^{*} e^{i\left(k_{3 p+i}-k_{2 p}\right) x}\right) e^{-i k_{p+i} x}, \\
& \frac{d A_{3 p}}{d x}=\frac{\beta}{2}\left(-k_{p} k_{2 p} A_{p} A_{2 p} e^{i\left(k_{p}+k_{2 p}\right) x}-k_{p+s} k_{p+i} A_{p+s} A_{p+i} e^{i\left(k_{p+s}+k_{p+i}\right) x}\right. \\
& -k_{s} k_{2 p+i} A_{s} A_{2 p+i} e^{i\left(k_{s}+k_{2 p+i}\right) x}-k_{i} k_{2 p+s} A_{i} A_{2 p+s} e^{i\left(k_{i}+k_{2 p+s}\right) x}+k_{4 p} k_{p} A_{4 p} A_{p}^{*} e^{i\left(k_{4 p}-k_{p}\right) x}+k_{3 p+s} k_{s} A_{3 p+s} A_{s}^{*} e^{i\left(k_{3 p+s}-k_{s}\right) x} \\
& \left.+k_{3 p+i} k_{i} A_{3 p+i} A_{i}^{*} e^{i\left(k_{3 p+i}-k_{i}\right) x}\right) e^{-i k_{3 p} x}, \\
& \frac{d A_{2 p+s}}{d x}=\frac{\beta}{2}\left(-k_{p} k_{p+s} A_{p} A_{p+s} e^{i\left(k_{p}+k_{p+s}\right) x}-k_{s} k_{2 p} A_{s} A_{2 p} e^{i\left(k_{s}+k_{2 p}\right) x}\right. \\
& \left.+k_{3 p} k_{i} A_{3 p} A_{i}^{*} e^{i\left(k_{3 p}-k_{i}\right) x}+k_{4 p} k_{p+i} A_{4 p} A_{p+i}^{*} e^{i\left(k_{4 p}-k_{p+i}\right) x}+k_{3 p+s} k_{p} A_{3 p+s} A_{p}^{*} e^{i\left(k_{3 p+s}-k_{p}\right) x}\right) e^{-i k_{2 p+s} x}, \\
& \frac{d A_{2 p+i}}{d x}=\frac{\beta}{2}\left(-k_{p} k_{p+i} A_{p} A_{p+i} e^{i\left(k_{p}+k_{p+i}\right) x}-k_{i} k_{2 p} A_{i} A_{2 p} e^{i\left(k_{i}+k_{2 p}\right) x}\right. \\
& \left.+k_{3 p} k_{s} A_{3 p} A_{s}^{*} e^{i\left(k_{3 p}-k_{s}\right) x}+k_{4 p} k_{p+s} A_{4 p} A_{p+s}^{*} e^{i\left(k_{4 p}-k_{p+s}\right) x}+k_{3 p+i} k_{p} A_{3 p+i} A_{p}^{*} e^{i\left(k_{3 p+i}-k_{p}\right) x}\right) e^{-i k_{2 p+i} x}, \\
& \frac{d A_{4 p}}{d x}=\frac{\beta}{2}\left(-k_{p} k_{3 p} A_{p} A_{3 p} e^{i\left(k_{p}+k_{3 p}\right) x}-k_{s} k_{3 p+i} A_{s} A_{3 p+i} e^{i\left(k_{3 p+i}+k_{s}\right) x}-k_{i} k_{3 p+s} A_{i} A_{3 p+s} e^{i\left(k_{i}+k_{3 p+s}\right) x}-\frac{k_{2 p}^{2} A_{2 p}^{2}}{2} e^{i\left(k_{2 p}+k_{2 p}\right) x}\right. \\
& \left.-k_{p+s} k_{2 p+i} A_{p+s} A_{2 p+i} e^{i\left(k_{p+s}+k_{2 p+i}\right) x}-k_{p+i} k_{2 p+s} A_{p+i} A_{2 p+s} e^{i\left(k_{p+i}+k_{2 p+s}\right) x}\right) e^{-i k_{4 p} x}, \\
& \frac{d A_{3 p+s}}{d x}=\frac{\beta}{2}\left(-k_{p} k_{2 p+s} A_{p} A_{2 p+s} e^{i\left(k_{p}+k_{2 p+s}\right) x}-k_{2 p} k_{p+s} A_{2 p} A_{p+s} e^{i\left(k_{2 p}+k_{p+s}\right) x}\right. \\
& \left.-k_{s} k_{3 p} A_{s} A_{3 p} e^{i\left(k_{s}+k_{3 p}\right) x}+k_{i} k_{4 p} A_{i}^{*} A_{4 p} e^{i\left(k_{4 p}-k_{i}\right) x}\right) e^{-i k_{3 p+s} x}, \\
& \frac{d A_{3 p+i}}{d x}=\frac{\beta}{2}\left(-k_{p} k_{2 p+i} A_{p} A_{2 p+i} e^{i\left(k_{p}+k_{2 p+i}\right) x}-k_{2 p} k_{p+i} A_{2 p} A_{p+i} e^{i\left(k_{2 p}+k_{p+i}\right) x}\right. \\
& \left.-k_{i} k_{3 p} A_{i} A_{3 p} e^{i\left(k_{i}+k_{3 p}\right) x}+k_{s} k_{4 p} A_{s}^{*} A_{4 p} e^{i\left(k_{4 p}-k_{s}\right) x}\right) e^{-i k_{3 p+i} x} .
\end{aligned}
$$

\section{APPENDIX G: EXTENSION TO CME-5}

The final coupled-mode-equation extension that we present in full is the extension from CME-4 to CME-5 by inclusion of the fifth harmonic of the pump $f_{5 p}$, and the sum-frequency terms $f_{4 p+i}$ and $f_{4 p+s}$. The full list of tones included in CME-5 is shown in Table I. We show below in detail all of the terms included in the coupled mode equations forming CME-5: 


$$
\begin{aligned}
& \frac{d A_{i}}{d x}=\frac{\beta}{2}\left(k_{s} k_{p} A_{p} A_{s}^{*} e^{i\left(k_{p}-k_{s}\right) x}+k_{p+i} k_{p} A_{p+i} A_{p}^{*} e^{i\left(k_{p+i}-k_{p}\right) x}\right. \\
& +k_{2 p} k_{p+s} A_{2 p} A_{p+s}^{*} e^{i\left(k_{2 p}-k_{p+s}\right) x}+k_{2 p+i} k_{2 p} A_{2 p+i} A_{2 p}^{*} e^{i\left(k_{2 p+i}-k_{2 p}\right) x} \\
& +k_{3 p} k_{2 p+s} A_{3 p} A_{2 p+s}^{*} e^{i\left(k_{3 p}-k_{2 p+s}\right) x}+k_{3 p+i} k_{3 p} A_{3 p+i} A_{3 p}^{*} e^{i\left(k_{3 p+i}-k_{3 p}\right) x} \\
& +k_{4 p} k_{3 p+s} A_{4 p} A_{3 p+s}^{*} e^{i\left(k_{4 p}-k_{3 p+s}\right) x}+k_{4 p+i} k_{4 p} A_{4 p+i} A_{4 p}^{*} e^{i\left(k_{4 p+i}-k_{4 p}\right) x} \\
& \left.+k_{5 p} k_{4 p+s} A_{5 p} A_{4 p+s}^{*} e^{i\left(k_{5 p}-k_{4 p+s}\right) x}\right) e^{-i k_{i} x}, \\
& \frac{d A_{s}}{d x}=\frac{\beta}{2}\left(k_{i} k_{p} A_{p} A_{i}^{*} e^{i\left(k_{p}-k_{i}\right) x}+k_{p+s} k_{p} A_{p+s} A_{p}^{*} e^{i\left(k_{p+s}-k_{p}\right) x}\right. \\
& +k_{2 p} k_{p+i} A_{2 p} A_{p+i}^{*} e^{i\left(k_{2 p}-k_{p+i}\right) x}+k_{2 p+s} k_{2 p} A_{2 p+s} A_{2 p}^{*} e^{i\left(k_{2 p+s}-k_{2 p}\right) x} \\
& +k_{3 p} k_{2 p+i} A_{3 p} A_{2 p+i}^{*} e^{i\left(k_{3 p}-k_{2 p+i}\right) x}+k_{3 p+s} k_{3 p} A_{3 p+s} A_{3 p}^{*} e^{i\left(k_{3 p+s}-k_{3 p}\right) x} \\
& +k_{4 p} k_{3 p+i} A_{4 p} A_{3 p+i}^{*} e^{i\left(k_{4 p}-k_{3 p+i}\right) x}+k_{4 p+s} k_{4 p} A_{4 p+s} A_{4 p}^{*} e^{i\left(k_{4 p+s}-k_{4 p}\right) x} \\
& \left.+k_{5 p} k_{4 p+i} A_{5 p} A_{4 p+i}^{*} e^{i\left(k_{5 p}-k_{4 p+i}\right) x}\right) e^{-i k_{s} x}, \\
& \frac{d A_{p}}{d x}=\frac{\beta}{2}\left(-k_{i} k_{s} A_{i} A_{s} e^{i\left(k_{i}+k_{s}\right) x}+k_{p+i} k_{i} A_{p+i} A_{i}^{*} e^{i\left(k_{p+i}-k_{i}\right) x}\right. \\
& +k_{p+s} k_{s} A_{p+s} A_{s}^{*} e^{i\left(k_{p+s}-k_{s}\right) x}+k_{2 p} k_{p} A_{2 p} A_{p}^{*} e^{i\left(k_{2 p}-k_{p}\right) x} \\
& +k_{2 p+i} k_{p+i} A_{2 p+i} A_{p+i}^{*} e^{i\left(k_{2 p+i}-k_{p+i}\right) x}+k_{2 p+s} k_{p+s} A_{2 p+s} A_{p+s}^{*} e^{i\left(k_{2 p+s}-k_{p+s}\right) x} \\
& +k_{3 p} k_{2 p} A_{3 p} A_{2 p}^{*} e^{i\left(k_{3 p}-k_{2 p}\right) x}+k_{3 p+i} k_{2 p+i} A_{3 p+i} A_{2 p+i}^{*} e^{i\left(k_{3 p+i}-k_{2 p+i}\right) x} \\
& +k_{3 p+s} k_{2 p+s} A_{3 p+s} A_{2 p+s}^{*} e^{i\left(k_{3 p+s}-k_{2 p+s}\right) x}+k_{4 p} k_{3 p} A_{4 p} A_{3 p}^{*} e^{i\left(k_{4 p}-k_{3 p}\right) x} \\
& +k_{4 p+i} k_{3 p+i} A_{4 p+i} A_{3 p+i}^{*} e^{i\left(k_{4 p+i}-k_{3 p+i}\right) x}+k_{4 p+s} k_{3 p+s} A_{4 p+s} A_{3 p+s}^{*} e^{i\left(k_{4 p+s}-k_{3 p+s}\right) x} \\
& \left.+k_{5 p} k_{4 p} A_{5 p} A_{4 p}^{*} e^{i\left(k_{5 p}-k_{4 p}\right) x}\right) e^{-i k_{p} x}, \\
& \frac{d A_{p+i}}{d x}=\frac{\beta}{2}\left(-k_{i} k_{p} A_{i} A_{p} e^{i\left(k_{i}+k_{p}\right) x}+k_{2 p} k_{s} A_{2 p} A_{s}^{*} e^{i\left(k_{2 p}-k_{s}\right) x}\right. \\
& +k_{2 p+i} k_{p} A_{2 p+i} A_{p}^{*} e^{i\left(k_{2 p+i}-k_{p}\right) x}+k_{3 p} k_{p+s} A_{3 p} A_{p+s}^{*} e^{i\left(k_{3 p}-k_{p+s}\right) x} \\
& +k_{3 p+i} k_{2 p} A_{3 p+i} A_{2 p}^{*} e^{i\left(k_{3 p+i}-k_{2 p}\right) x}+k_{4 p} k_{2 p+s} A_{4 p} A_{2 p+s}^{*} e^{i\left(k_{4 p}-k_{2 p+s}\right) x} \\
& \left.+k_{4 p+i} k_{3 p} A_{4 p+i} A_{3 p}^{*} e^{i\left(k_{4 p+i}-k_{3 p}\right) x}+k_{5 p} k_{3 p+s} A_{5 p} A_{3 p+s}^{*} e^{i\left(k_{5 p}-k_{3 p+s}\right) x}\right) e^{-i k_{p+i} x} \text {, } \\
& \frac{d A_{p+s}}{d x}=\frac{\beta}{2}\left(-k_{s} k_{p} A_{s} A_{p} e^{i\left(k_{s}+k_{p}\right) x}+k_{2 p} k_{i} A_{2 p} A_{i}^{*} e^{i\left(k_{2 p}-k_{i}\right) x}\right. \\
& +k_{2 p+s} k_{p} A_{2 p+s} A_{p}^{*} e^{i\left(k_{2 p+s}-k_{p}\right) x}+k_{3 p} k_{p+i} A_{3 p} A_{p+i}^{*} e^{i\left(k_{3 p}-k_{p+i}\right) x} \\
& +k_{3 p+s} k_{2 p} A_{3 p+s} A_{2 p}^{*} e^{i\left(k_{3 p+s}-k_{2 p}\right) x}+k_{4 p} k_{2 p+i} A_{4 p} A_{2 p+i}^{*} e^{i\left(k_{4 p}-k_{2 p+i}\right) x} \\
& \left.+k_{4 p+s} k_{3 p} A_{4 p+s} A_{3 p}^{*} e^{i\left(k_{4 p+s}-k_{3 p}\right) x}+k_{5 p} k_{3 p+i} A_{5 p} A_{3 p+i}^{*} e^{i\left(k_{5 p}-k_{3 p+i}\right) x}\right) e^{-i k_{p+s} x}, \\
& \frac{d A_{2 p}}{d x}=\frac{\beta}{2}\left(-k_{i} k_{p+s} A_{i} A_{p+s} e^{i\left(k_{i}+k_{p+s}\right) x}-k_{s} k_{p+i} A_{s} A_{p+i} e^{i\left(k_{s}+k_{p+i}\right) x}\right. \\
& -\frac{k_{p}^{2} A_{p}^{2}}{2} e^{i\left(k_{p}+k_{p}\right) x}+k_{2 p+i} k_{i} A_{2 p+i} A_{i}^{*} e^{i\left(k_{2 p+i}-k_{i}\right) x} \\
& +k_{2 p+s} k_{s} A_{2 p+s} A_{s}^{*} e^{i\left(k_{2 p+s}-k_{s}\right) x}+k_{3 p} k_{p} A_{3 p} A_{p}^{*} e^{i\left(k_{3 p}-k_{p}\right) x}
\end{aligned}
$$




$$
\begin{aligned}
& +k_{3 p+i} k_{p+i} A_{3 p+i} A_{p+i}^{*} e^{i\left(k_{3 p+i}-k_{p+i}\right) x}+k_{3 p+s} k_{p+s} A_{3 p+s} A_{p+s}^{*} e^{i\left(k_{3 p+s}-k_{p+s}\right) x} \\
& +k_{4 p} k_{2 p} A_{4 p} A_{2 p}^{*} e^{i\left(k_{4 p}-k_{2 p}\right) x}+k_{4 p+i} k_{2 p+i} A_{4 p+i} A_{2 p+i}^{*} e^{i\left(k_{4 p+i}-k_{2 p+i}\right) x} \\
& \left.+k_{4 p+s} k_{2 p+s} A_{4 p+s} A_{2 p+s}^{*} e^{i\left(k_{4 p+s}-k_{2 p+s}\right) x}+k_{5 p} k_{3 p} A_{5 p} A_{3 p}^{*} e^{i\left(k_{5 p}-k_{3 p}\right) x}\right) e^{-i k_{2 p} x}, \\
& \frac{d A_{2 p+i}}{d x}=\frac{\beta}{2}\left(-k_{i} k_{2 p} A_{i} A_{2 p} e^{i\left(k_{i}+k_{2 p}\right) x}-k_{p} k_{p+i} A_{p} A_{p+i} e^{i\left(k_{p}+k_{p+i}\right) x}\right. \\
& +k_{3 p} k_{s} A_{3 p} A_{s}^{*} e^{i\left(k_{3 p}-k_{s}\right) x}+k_{3 p+i} k_{p} A_{3 p+i} A_{p}^{*} e^{i\left(k_{3 p+i}-k_{p}\right) x} \\
& +k_{4 p} k_{p+s} A_{4 p} A_{p+s}^{*} e^{i\left(k_{4 p}-k_{p+s}\right) x}+k_{4 p+i} k_{2 p} A_{4 p+i} A_{2 p}^{*} e^{i\left(k_{4 p+i}-k_{2 p}\right) x} \\
& \left.+k_{5 p} k_{2 p+s} A_{5 p} A_{2 p+s}^{*} e^{i\left(k_{5 p}-k_{2 p+s}\right) x}\right) e^{-i k_{2 p+i} x}, \\
& \frac{d A_{2 p+s}}{d x}=\frac{\beta}{2}\left(-k_{s} k_{2 p} A_{s} A_{2 p} e^{i\left(k_{s}+k_{2 p}\right) x}-k_{p} k_{p+s} A_{p} A_{p+s} e^{i\left(k_{p}+k_{p+s}\right) x}\right. \\
& +k_{3 p} k_{i} A_{3 p} A_{i}^{*} e^{i\left(k_{3 p}-k_{i}\right) x}+k_{3 p+s} k_{p} A_{3 p+s} A_{p}^{*} e^{i\left(k_{3 p+s}-k_{p}\right) x} \\
& +k_{4 p} k_{p+i} A_{4 p} A_{p+i}^{*} e^{i\left(k_{4 p}-k_{p+i}\right) x}+k_{4 p+s} k_{2 p} A_{4 p+s} A_{2 p}^{*} e^{i\left(k_{4 p+s}-k_{2 p}\right) x} \\
& \left.+k_{5 p} k_{2 p+i} A_{5 p} A_{2 p+i}^{*} e^{i\left(k_{5 p}-k_{2 p+i}\right) x}\right) e^{-i k_{2 p+s} x}, \\
& \frac{d A_{3 p}}{d x}=\frac{\beta}{2}\left(-k_{i} k_{2 p+s} A_{i} A_{2 p+s} e^{i\left(k_{i}+k_{2 p+s}\right) x}-k_{s} k_{2 p+i} A_{s} A_{2 p+i} e^{i\left(k_{s}+k_{2 p+i}\right) x}\right. \\
& -k_{p} k_{2 p} A_{p} A_{2 p} e^{i\left(k_{p}+k_{2 p}\right) x}-k_{p+i} k_{p+s} A_{p+i} A_{p+s} e^{i\left(k_{p+i}+k_{p+s}\right) x} \\
& +k_{3 p+i} k_{i} A_{3 p+i} A_{i}^{*} e^{i\left(k_{3 p+i}-k_{i}\right) x}+k_{3 p+s} k_{s} A_{3 p+s} A_{s}^{*} e^{i\left(k_{3 p+s}-k_{s}\right) x} \\
& +k_{4 p} k_{p} A_{4 p} A_{p}^{*} e^{i\left(k_{4 p}-k_{p}\right) x}+k_{4 p+i} k_{p+i} A_{4 p+i} A_{p+i}^{*} e^{i\left(k_{4 p+i}-k_{p+i}\right) x} \\
& \left.+k_{4 p+s} k_{p+s} A_{4 p+s} A_{p+s}^{*} e^{i\left(k_{4 p+s}-k_{p+s}\right) x}+k_{5 p} k_{2 p} A_{5 p} A_{2 p}^{*} e^{i\left(k_{5 p}-k_{2 p}\right) x}\right) e^{-i k_{3 p} x}, \\
& \frac{d A_{3 p+i}}{d x}=\frac{\beta}{2}\left(-k_{i} k_{3 p} A_{i} A_{3 p} e^{i\left(k_{i}+k_{3 p}\right) x}-k_{p} k_{2 p+i} A_{p} A_{2 p+i} e^{i\left(k_{p}+k_{2 p+i}\right) x}\right. \\
& -k_{p+i} k_{2 p} A_{p+i} A_{2 p} e^{i\left(k_{p+i}+k_{2 p}\right) x}+k_{4 p} k_{s} A_{4 p} A_{s}^{*} e^{i\left(k_{4 p}-k_{s}\right) x} \\
& \left.+k_{4 p+i} k_{p} A_{4 p+i} A_{p}^{*} e^{i\left(k_{4 p+i}-k_{p}\right) x}+k_{5 p} k_{p+s} A_{5 p} A_{p+s}^{*} e^{i\left(k_{5 p}-k_{p+s}\right) x}\right) e^{-i k_{3 p+i} x}, \\
& \frac{d A_{3 p+s}}{d x}=\frac{\beta}{2}\left(-k_{s} k_{3 p} A_{s} A_{3 p} e^{i\left(k_{s}+k_{3 p}\right) x}-k_{p} k_{2 p+s} A_{p} A_{2 p+s} e^{i\left(k_{p}+k_{2 p+s}\right) x}\right. \\
& -k_{p+s} k_{2 p} A_{p+s} A_{2 p} e^{i\left(k_{p+s}+k_{2 p}\right) x}+k_{4 p} k_{i} A_{4 p} A_{i}^{*} e^{i\left(k_{4 p}-k_{i}\right) x} \\
& \left.+k_{4 p+s} k_{p} A_{4 p+s} A_{p}^{*} e^{i\left(k_{4 p+s}-k_{p}\right) x}+k_{5 p} k_{p+i} A_{5 p} A_{p+i}^{*} e^{i\left(k_{5 p}-k_{p+i}\right) x}\right) e^{-i k_{3 p+s} x}, \\
& \frac{d A_{4 p}}{d x}=\frac{\beta}{2}\left(-k_{i} k_{3 p+s} A_{i} A_{3 p+s} e^{i\left(k_{i}+k_{3 p+s}\right) x}-k_{s} k_{3 p+i} A_{S} A_{3 p+i} e^{i\left(k_{s}+k_{3 p+i}\right) x}\right. \\
& -k_{p} k_{3 p} A_{p} A_{3 p} e^{i\left(k_{p}+k_{3 p}\right) x}-k_{p+i} k_{2 p+s} A_{p+i} A_{2 p+s} e^{i\left(k_{p+i}+k_{2 p+s}\right) x} \\
& -k_{p+s} k_{2 p+i} A_{p+s} A_{2 p+i} e^{i\left(k_{p+s}+k_{2 p+i}\right) x}-\frac{k_{2 p}^{2} A_{2 p}^{2}}{2} e^{i\left(k_{2 p}+k_{2 p}\right) x} \\
& +k_{4 p+i} k_{i} A_{4 p+i} A_{i}^{*} e^{i\left(k_{4 p+i}-k_{i}\right) x}+k_{4 p+s} k_{s} A_{4 p+s} A_{s}^{*} e^{i\left(k_{4 p+s}-k_{s}\right) x} \\
& \left.+k_{5 p} k_{p} A_{5 p} A_{p}^{*} e^{i\left(k_{5 p}-k_{p}\right) x}\right) e^{-i k_{4 p} x},
\end{aligned}
$$




$$
\begin{aligned}
\frac{d A_{4 p+i}}{d x}= & \frac{\beta}{2}\left(-k_{i} k_{4 p} A_{i} A_{4 p} e^{i\left(k_{i}+k_{4 p}\right) x}-k_{p} k_{3 p+i} A_{p} A_{3 p+i} e^{i\left(k_{p}+k_{3 p+i}\right) x}\right. \\
& -k_{p+i} k_{3 p} A_{p+i} A_{3 p} e^{i\left(k_{p+i}+k_{3 p}\right) x}-k_{2 p} k_{2 p+i} A_{2 p} A_{2 p+i} e^{i\left(k_{2 p}+k_{2 p+i}\right) x} \\
& \left.+k_{5 p} k_{s} A_{5 p} A_{s}^{*} e^{i\left(k_{5 p}-k_{s}\right) x}\right) e^{-i k_{4 p+i} x} \\
\frac{d A_{4 p+s}}{d x}= & \frac{\beta}{2}\left(-k_{s} k_{4 p} A_{s} A_{4 p} e^{i\left(k_{s}+k_{4 p}\right) x}-k_{p} k_{3 p+s} A_{p} A_{3 p+s} e^{i\left(k_{p}+k_{3 p+s}\right) x}\right. \\
& -k_{p+s} k_{3 p} A_{p+s} A_{3 p} e^{i\left(k_{p+s}+k_{3 p}\right) x}-k_{2 p} k_{2 p+s} A_{2 p} A_{2 p+s} e^{i\left(k_{2 p}+k_{2 p+s}\right) x} \\
& \left.+k_{5 p} k_{i} A_{5 p} A_{i}^{*} e^{i\left(k_{5 p}-k_{i}\right) x}\right) e^{-i k_{4 p+s} x}, \\
\frac{d A_{5 p}}{d x}= & \frac{\beta}{2}\left(-k_{i} k_{4 p+s} A_{i} A_{4 p+s} e^{i\left(k_{i}+k_{4 p+s}\right) x}-k_{s} k_{4 p+i} A_{s} A_{4 p+i} e^{i\left(k_{s}+k_{4 p+i}\right) x}\right. \\
& -k_{p} k_{4 p} A_{p} A_{4 p} e^{i\left(k_{p}+k_{4 p}\right) x}-k_{p+i} k_{3 p+s} A_{p+i} A_{3 p+s} e^{i\left(k_{p+i}+k_{3 p+s}\right) x} \\
& -k_{p+s} k_{3 p+i} A_{p+s} A_{3 p+i} e^{i\left(k_{p+s}+k_{3 p+i}\right) x}-k_{2 p} k_{3 p} A_{2 p} A_{3 p} e^{i\left(k_{2 p}+k_{3 p}\right) x} \\
& \left.-k_{2 p+i} k_{2 p+s} A_{2 p+i} A_{2 p+s} e^{i\left(k_{2 p+i}+k_{2 p+s}\right) x}\right) e^{-i k_{5 p} x} .
\end{aligned}
$$

[1] B. Yurke, L. R. Corruccini, P. G. Kaminsky, L. W. Rupp, A. D. Smith, A. H. Silver, R. W. Simon, and E. A. Whittaker, Observation of parametric amplification and deamplification in a Josephson parametric amplifier, Phys. Rev. A 39, 2519 (1989).

[2] T. Yamamoto, K. Inomata, M. Watanabe, K. Matsuba, T. Miyazaki, W. D. Oliver, Y. Nakamura, and J. S. Tsai, Fluxdriven Josephson parametric amplifier, Appl. Phys. Lett. 93, 042510 (2008).

[3] J. Y. Mutus, T. C. White, R. Barends, Yu Chen, Z. Chen, B. Chiaro, A. Dunsworth, E. Jeffrey, J. Kelly, A. Megrant, C. Neill, P. J. J. O’Malley, P. Roushan, D. Sank, A. Vainsencher, J. Wenner, K. M. Sundqvist, A. N. Cleland, and J. M. Martinis, Strong environmental coupling in a Josephson parametric amplifier, Appl. Phys. Lett. 104, 263513 (2014).

[4] F. Mallet, M. A. Castellanos-Beltran, H. S. Ku, S. Glancy, E. Knill, K. D. Irwin, G. C. Hilton, L. R. Vale, and K. W. Lehnert, Quantum State Tomography of an Itinerant Squeezed Microwave Field, Phys. Rev. Lett. 106, 220502 (2011).

[5] W. F. Kindel, M. D. Schroer, and K. W. Lehnert, Generation and efficient measurement of single photons from fixedfrequency superconducting qubits, Phys. Rev. A 93, 033817 (2016).

[6] S. Hacohen-Gourgy, L. P. García-Pintos, L. S. Martin, J. Dressel, and I. Siddiqi, Incoherent Qubit Control Using the Quantum Zeno Effect, Phys. Rev. Lett. 120, 020505 (2018).

[7] Z. R. Lin, K. Inomata, W. D. Oliver, K. Koshino, Y. Nakamura, J. S. Tsai, and T. Yamamoto, Single-shot readout of a superconducting flux qubit with a flux-driven Josephson parametric amplifier, Appl. Phys. Lett. 103, 132602 (2013).
[8] M. A. Castellanos-Beltran, K. D. Irwin, G. C. Hilton, L. R. Vale, and K. W. Lehnert, Amplification and squeezing of quantum noise with a tunable Josephson metamaterial, Nat. Phys. 4, 929 (2008).

[9] M. A. Castellanos-Beltran and K. W. Lehnert, Widely tunable parametric amplifier based on a superconducting quantum interference device array resonator, Appl. Phys. Lett. 91, 083509 (2007).

[10] X. Zhou, V. Schmitt, P. Bertet, D. Vion, W. Wustmann, V. Shumeiko, and D. Esteve, High-gain weakly nonlinear fluxmodulated Josephson parametric amplifier using a squid array, Phys. Rev. B 89, 214517 (2014).

[11] J. D. Teufel, T. Donner, M. A. Castellanos-Beltran, J. W. Harlow, and K. W. Lehnert, Nanomechanical motion measured with an imprecision below that at the standard quantum limit, Nat. Nanotechnol. 4, 820 (2009).

[12] B. H. Eom, P. K. Day, H. G. LeDuc, and J. Zmuidzinas, A wideband, low-noise superconducting amplifier with high dynamic range, Nat. Phys. 8, 623 (2012).

[13] C. Macklin, K. O’Brien, D. Hover, M. E. Schwartz, V. Bolkhovsky, X. Zhang, W. D. Oliver, and I. Siddiqi, A near-quantum-limited Josephson traveling-wave parametric amplifier, Science 350, 307 (2015).

[14] T. C. White et al., Traveling wave parametric amplifier with Josephson junctions using minimal resonator phase matching, Appl. Phys. Lett. 106, 242601 (2015).

[15] O. Yaakobi, L. Friedland, C. Macklin, and I. Siddiqi, Parametric amplification in Josephson junction embedded transmission lines, Phys. Rev. B 87, 144301 (2013).

[16] A. B. Zorin, Josephson Traveling-Wave Parametric Amplifier with Three-Wave Mixing, Phys. Rev. Appl. 6, 034006 (2016).

[17] S. R. Whiteley, Josephson junctions in spice3, IEEE 23S, 2902 (1991), https://ieeexplore.ieee.org/document/133816. 
[18] A. L. Cullen, Theory of the travelling-wave parametric amplifier, Proc. IEE - Part B: Electron. Commun. Eng. 107, 101 (1960).

[19] S. Chaudhuri, J. Gao, and K. Irwin, Simulation and analysis of superconducting traveling-wave parametric amplifiers, IEEE Trans. Appl. Supercond. 25, 1 (2015).

[20] A. B. Zorin, Flux-Driven Josephson Traveling-Wave Parametric Amplifier, Phys. Rev. Appl. 12, 044051 (2019).
[21] R. P. Erickson and D. P. Pappas, Theory of multiwave mixing within the superconducting kinetic-inductance traveling-wave amplifier, Phys. Rev. B 95, 104506 (2017).

[22] C. D. Shelly, T. Dixon, J. W. Dunstan, G. B. Long, J. M. Williams, and P. J. Meeson, JTWPA Circuit - WRspice (2020), https://doi.org/10.5281/zenodo.3948306.

[23] A. K. Walton, Network Analysis and Practice (Cambridge University Press, New York, 1987). 